\title{
RANDOM GROUPS AND NONARCHIMEDEAN LATTICES
}

\author{
SYLVAIN BARRÉ ${ }^{1}$ and MIKAËL PICHOT ${ }^{2}$ \\ ${ }^{1}$ Université de Bretagne Sud, Université Européenne de Bretagne, France; \\ email: sylvain.barre@univ.ubs.fr \\ ${ }^{2}$ Department of Mathematics \& Statistics, McGill University, Montréal, Québec, \\ Canada H3A 2K6; \\ email: pichot@math.mcgill.ca
}

Received 22 January 2014; accepted 29 August 2014

\begin{abstract}
We consider models of random groups in which the typical group is of intermediate rank (in particular, it is not hyperbolic). These models are parallel to Gromov's well-known constructions, and include for example a 'density model' for groups of intermediate rank. The main novelty is the higher rank nature of the random groups. They are randomizations of certain families of lattices in algebraic groups (of rank 2) over local fields.
\end{abstract}

2010 Mathematics Subject Classification: 20F65 (primary); 20E42, 20E18 (secondary)

\section{Introduction}

This paper introduces models of random groups 'of higher rank'. The construction, basic properties, and applications are detailed in $\S 2$ to $\S 8$ below, which we now summarize.

The construction (see $\S 2$ ) is rather general. If $\Gamma^{\prime}$ is a group which acts properly on a simply connected complex $X^{\prime}$ of dimension 2 with $X^{\prime} / \Gamma^{\prime}$ compact, and $\Gamma^{\prime \prime} \subset$ $\Gamma^{\prime}$ is a subgroup of 'very large' finite index, then one can choose at random a family of $\Gamma^{\prime \prime}$-orbits of 2-cells $Y \subset X^{\prime}$ inside $X^{\prime}$. Then let $X$ denote the universal cover of $X^{\prime \prime}:=X^{\prime} \backslash Y$. The random group $\Gamma$ is the group of transformations of the Galois covering

$$
X \rightarrow X^{\prime \prime} / \Gamma^{\prime \prime} \text {. }
$$

(c) The Author(s) 2014. This is an Open Access article, distributed under the terms of the Creative Commons Attribution licence (http://creativecommons.org/licenses/by/3.0/), which permits unrestricted re-use, distribution, and reproduction in any medium, provided the original work is properly cited. 
This construction leads to several distinct models of random groups including a 'density model', following Gromov. The initial structural data $\left(\Gamma^{\prime}, X^{\prime}, \ldots\right)$ for the model is called the deterministic data. The basic properties of $\Gamma$ depend on the deterministic data.

An idea of groups 'of intermediate rank' was introduced in [2] in particular to address the following question, where $X$ is $\operatorname{CAT}(0)$ and $X / \Gamma$ is compact:

$$
\mathbb{R}^{2} \hookrightarrow X \Rightarrow \mathbb{Z}^{2} \hookrightarrow \Gamma ?
$$

(This is the 'periodic flat plane problem' which has been formulated in many places; see [12] for an early reference.) Since the assumption $\mathbb{R}^{2} \hookrightarrow X$ is equivalent to $X$ being nonhyperbolic, the new models are relevant to the study of this question. We will see that in some cases (depending on the deterministic data, the density parameter, etc.) the answer is positive 'generically', but that the precise relation between the two conditions ' $\mathbb{R}^{2} \hookrightarrow X$ ' and ' $\mathbb{Z}^{2} \hookrightarrow \Gamma$ ' remains mysterious even for random groups associated with lattices in $\mathrm{PSL}_{3}$.

Before turning to these models, let us discuss briefly Gromov's original construction of random groups and the density model introduced in [14] (see also [12, Section 6], [13], or [15]).

Let $\Gamma_{1}$ be a hyperbolic group in the sense of Gromov, and take successive quotients $\Gamma_{1} \rightarrow \Gamma_{2} \rightarrow \cdots$, say

$$
\Gamma_{n+1}:=\Gamma_{n} /\left\langle\left\langle R_{n}\right\rangle\right\rangle
$$

where $R_{n} \subset \Gamma_{n}$ is a finite set of additional relations. As explained in [14], the set $R_{n}$ can 'in general' be chosen so that the following hold:

(i) $\Gamma_{n+1}$ stays hyperbolic;

(ii) $\Gamma_{n} \rightarrow \Gamma_{n+1}$ is injective on larger and larger balls.

Property (ii) ensures the existence of an infinite limit group $\Gamma_{\infty}$, while the attribute 'in general' accounts for the oversupply of choices in the construction; its precise meaning depends on the size and the nature of $R_{n}$. For example, if $\Gamma_{1}$ is torsion free and the sets $R_{n}$ consist of a single relation which is a 'higher and higher' power of the $n$th element in a list exhausting $\Gamma_{1}$, then the limit $\Gamma_{\infty}$ is a finitely generated infinite torsion group [14, Section 4.5.C]. Here, (i) and (ii) become geometric assertions relying on $K<0$, and the construction offers almost total freedom. (Gromov's construction is related to the Burnside problem-the existence of infinite torsion groups was established by Golod, and the existence of infinite groups of finite exponent by Adian and Novikov, and by Olshanskii using the small cancellation theory.) 
A prominent feature is the genericity of hyperbolic groups, as put forward in [14] and illustrated by (i) above. Gromov has since invented several models of random groups and constructed many exotic infinite groups using them [11, 15$17,21]$. We are interested here in his so-called density model, which studies 'onestep' random quotients $\Gamma_{n} \rightarrow \Gamma_{n+1}$ for 'very large' random sets $R_{n}$ of 'very long' relations. If one starts with a free group $F_{r}$ on $r$ generators, and we let $\delta$ denote the density parameter, then the random group in the density model is a quotient of the form $F_{r} /\left\langle\left\langle W_{p}\right\rangle\right\rangle$, where $W_{p}$ is a set of $\approx\left|S_{p}\right|^{\delta}$ words chosen uniformly independently at random in the sphere $S_{p}$ of radius $p$ in $F_{r}$. Gromov shows that, if $\delta<1 / 2$, then the resulting random group is hyperbolic with overwhelming probability as $p \rightarrow \infty$, while if $\delta>1 / 2$ it is trivial (meaning 1 or \pm ) with overwhelming probability. In this model, small cancellations occur for $\delta<1 / 12$. (One can also start here with a nonelementary hyperbolic group $\Gamma$ and take random quotients by elements in the spheres $S_{p} \subset \Gamma, p \rightarrow \infty$; the same phase transition ' $\delta<1 / 2 \Rightarrow$ hyperbolic' and ' $\delta>1 / 2 \Rightarrow 1 / \pm$ ' is then valid provided that $\Gamma$ is torsion free [22].) An earlier model of Gromov, called the 'few-relator model', studies the situation where $\left|W_{p}\right|$ is bounded. We refer to [21] for a survey of these groups.

The groups of intermediate rank constructed in [2] can be put on a 'rank interpolation line':

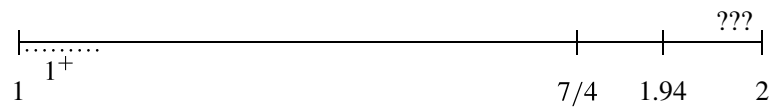

(we emphasize again that this only has a schematic value: as discussed in [2], the phenomenon of rank interpolation is not unidimensional.) The two extreme cases in this picture are the hyperbolic groups (rank 1, or more generally the groups with isolated flats, of rank $1^{+}$) and the lattices in nonarchimedean groups (rank 2). The value 1.94 refers to the bowtie group $\Gamma_{\bowtie}$ introduced in [2] and further studied in [4]. The present paper constructs many groups whose rank is arbitrarily close to 2: if the deterministic data arises from a nonarchimedean Lie group of rank 2, then the 'rank' of the random group (for example, the local rank in the sense of [3, Definition 4.5]) is as close to 2 as desired.

We now formulate our main result in the special case of the density model with deterministic data the Cartwright-Steger lattices in $\operatorname{PGL}_{3}\left(\mathbf{F}_{q}((y))\right)$ and their congruence subgroups. The techniques and constructions involved in the proof of this result apply in more general situations, and we will state and establish more general statements along the text. In fact, most of the assertions in Theorem 1, with the notable exception of the fact that ' $\delta<\frac{5}{8} \Rightarrow \mathbb{Z}^{2} \hookrightarrow \Gamma^{\prime}$ ', will be proved under less restrictive assumptions. 
The Cartwright-Steger lattices [7] are uniform lattices $\Gamma_{n}<\operatorname{PGL}_{n}\left(\mathbf{F}_{q}((y))\right)$ associated with the ring $R=\mathbf{F}_{q}[y, 1 / y, 1 /(1+y)] \hookrightarrow \mathbf{F}_{q}(y)$. Their congruence subgroups $\Gamma_{n}(I)$ correspond to ideals $I \triangleleft R$. The groups $\Gamma_{n}$ act transitively on the vertexes of the Bruhat-Tits building $X_{n}$ of $\operatorname{PGL}_{n}\left(\mathbf{F}_{q}((y))\right)$. Below $n=3$; the random groups discussed in Theorem 1 have as deterministic data the lattices $\left(X_{3}, \Gamma_{3},\left\{\Gamma_{3}\left(I_{p}\right)\right\}\right)$ associated with the Cartwright-Steger lattices of rank 2.

THEOREM 1. Let $q$ be a prime power. Fix two sequences $\left(f_{p}\right)_{p \geqslant 1}$ and $\left(s_{p}\right)_{p \geqslant 1}$, where $f_{p} \in \mathbf{F}_{q}[y]$ is a monic irreducible polynomial prime to $y$ and $y+1$ and $s_{p} \geqslant 1$ is an integer. If the density parameter $\delta$ satisfies

$$
\delta<\frac{5}{8}
$$

then the random group $\Gamma$ in the density model of parameter $\delta$ with (CartwrightSteger) deterministic data $\left(X_{3}, \Gamma_{3},\left\{\Gamma_{3}\left(I_{p}\right)\right\}\right)$ satisfies

$$
\mathbb{Z}^{2} \hookrightarrow \Gamma
$$

with overwhelming probability, where the congruence subgroups $\Gamma_{3}\left(I_{p}\right)_{p \geqslant 1}$ are associated with the ideal $I_{p}=\left\langle f_{p}^{s_{p}}\right\rangle$ generated by $f_{p}^{s_{p}}$ in $\mathbf{F}_{q}[y, 1 / y, 1 /(1+y)]$. If in addition $s_{p} \geqslant k$ for $p$ large enough, then $\mathbb{Z}^{2} \hookrightarrow \Gamma$ with overwhelming probability whenever

$$
\delta<\frac{7 k-3}{7 k+1}
$$

(which can be made as close to 1 as desired, independently of $q$ ). Furthermore, if

$$
\delta<\frac{q-1}{q-2}
$$

(which can be made as close to 1 as desired, independently of $k$ ), then $\Gamma$ acts freely uniformly on a space $X$ of dimension 2 with the geodesic extension property, and if

$$
\delta<\frac{1}{2} \text { and } q \geqslant 5
$$

then $\Gamma$ has Kazhdan's property $T$ with overwhelming probability. In addition, if $\delta_{0}$ is an arbitrary real number $<1$ given in advance, then there exists $q_{0}$ such that, if $q \geqslant q_{0}$, then $\Gamma$ has Kazhdan's property $T$ with overwhelming probability for every $\delta<\delta_{0}$. If, on the other hand,

$$
\delta>\frac{q}{q-1},
$$

then $\Gamma$ does not have property FA. Finally, if

$$
\delta<\frac{1}{2}
$$


(and $q$ is arbitrary), then $(X, \Gamma)$ has the extension rigidity property of [3] (namely, it 'remembers' the building $X_{3}$ it comes from). Finally, if $\delta_{0}<1$ and $\varepsilon>0$ are real numbers given in advance, then there exists $q_{0}$ such that, if $q \geqslant q_{0}$, then $(X, \Gamma)$ has local rank (in the sense of [3]) uniformly $\geqslant 2-\varepsilon$ with overwhelming probability for every $\delta<\delta_{0}$.

The paper is structured as follows. An analog of Gromov's few-relator model is studied in Section 4 in relation with the periodic flat plane problem. The density model is studied from Section 5 onwards, where we introduce 'critical densities' for various properties of groups in this model. In our framework, the density parameter $\delta$ regulates the size of the random subset $Y \subset X^{\prime}$. For example, we have a critical density $\delta_{T}$ for Kazhdan's property T, $\delta_{F A}$ for property FA, etc., and most importantly the critical density $\delta_{\mathbb{Z}^{2}}$ for the property that $\mathbb{Z}^{2} \hookrightarrow \Gamma$. The critical densities depend on the deterministic data. In Section 6, we discuss various analogs of Gromov's $\delta=1 / 2$ phase transition theorem in the density model, while Section 7 is devoted to estimating $\delta_{\mathbb{Z}^{2}}$ for nonarchimedean lattices (in positive characteristic). Finally, Section 8 derives other properties of the random group (for example property $\mathrm{T}$ ) and its 'intermediate rank' behavior. The reason why property $\mathrm{T}$ arises only 'for sufficiently large residue fields' is the same as in Garland's paper [10], namely that the spectral gap is large enough only for $q$ large enough.

We conclude with a question, the issue of which seems hard to predict at this stage.

QUESTION 2. Is there $(X, \Gamma)$ of local rank $>r$ such that $\mathbb{Z}^{2} \hookrightarrow \Gamma$ for every $r<2$ ?

Here, $X$ is a CAT( 0$)$ of dimension 2 and $\Gamma \curvearrowright X$ freely with $X / \Gamma$ compact, and the local rank is defined in [3]. The question can be considered both in the general case or when the order $q$ is bounded. See [3, Question 0.2] for a related question (some aspects of the 'local to global problem' implicit in Question 2 are also discussed in [3]) and also [4, Problem 3].

\section{Description of the random group}

Let $\Gamma$ be a discrete group acting freely simplicially on a 2-complex $X$ with $X / \Gamma$ compact, and let $\Gamma_{1}, \Gamma_{2}, \ldots$ be a family of finite index subgroups of $\Gamma$ with $\left[\Gamma: \Gamma_{p}\right] \rightarrow \infty, p \rightarrow \infty$. The random group defined below is a 'randomization' of the following (deterministic) sequence of covering maps: 


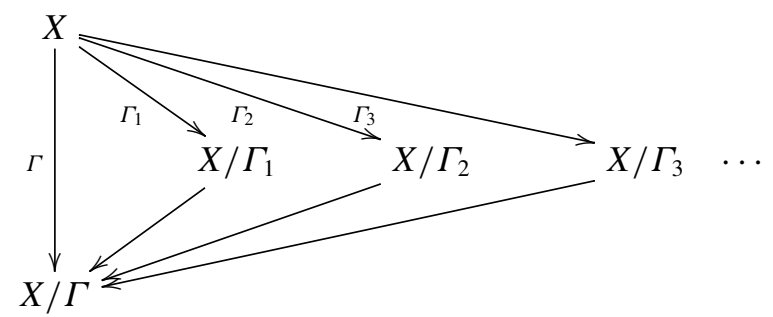

associated with $X$ and $\left(\Gamma_{p}\right)_{p \geqslant 1}$. (The family may be nested $\Gamma \geqslant \Gamma_{1} \geqslant \Gamma_{2} \cdots$, and may correspond to a tower $X / \Gamma \leftarrow X / \Gamma_{1} \leftarrow X / \Gamma_{2} \leftarrow X / \Gamma_{3} \leftarrow \cdots$ of compact spaces.) The randomization is achieved by inserting a 'random topological noise' to the spaces $X / \Gamma_{p}$ (where $p$ is very large) which is detected by the fundamental group.

Definition 3. We call $\left(X, \Gamma,\left\{\Gamma_{p}\right\}\right)$ the deterministic data.

The topological perturbation is implemented as follows. For each $p \geqslant 1$, remove a family of 2-cells in $X / \Gamma_{p}$ (equivalently, a family of $\Gamma_{p}$-orbits of 2-cells in the fixed space $X$ ) at random (with respect to a probability scheme for removing 2-cells, for example, Bernoulli). The universal cover $\tilde{X}_{p}$ of the resulting (random) space $X_{p} \subset X$ has a (random) group $\tilde{\Gamma}_{p}$ of deck transformations:

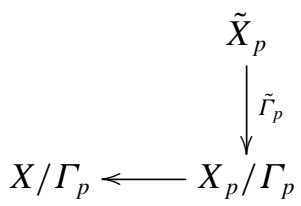

as described in the following overall diagram:

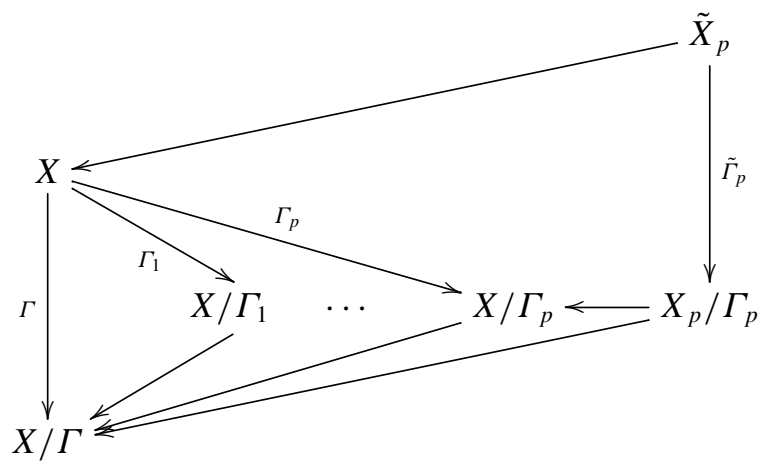


We study the properties of $\left(\tilde{X}_{p}, \tilde{\Gamma}_{p}\right)$ when the order of approximation $p$ is very large. The construction provides a random group $\tilde{\Gamma}_{p}$, a random space $\tilde{X}_{p}$, a random action $\tilde{\Gamma}_{p} \curvearrowright \tilde{X}_{p}$, and a random compact space $\tilde{X}_{p} / \tilde{\Gamma}_{p}$.

At this stage, the model will be precisely defined as long as the random scheme removing 2-cells is specified; several options are possible. We study here analogs of Gromov's well-known models for adding relators at random to a finitely generated group (more precisely, analogs of the 'few-relator model' and the 'density model' of $[14,15])$.

Fix the deterministic data $\left(X, \Gamma,\left\{\Gamma_{p}\right\}\right)$ (different choices for the triples $(X, \Gamma$, $\left.\left\{\Gamma_{p}\right\}\right)$ give rise to different models of random groups and lead to a priori distinct random objects). The set

$$
\mathscr{C}_{p}:=\left\{\Gamma_{p} \text {-orbits of faces in } X\right\}
$$

(whose element are called equivariant faces of $X$ with respect to $\Gamma_{p}$, or sometimes equivariant chambers when we have in mind a Bruhat-Tits building) is finite, and in many interesting cases it is rapidly growing. It plays the role of the set

$$
W_{p}:=\text { all words (or reduced words) of length } p \text { (or at most } p \text { ) in } \Gamma
$$

in Gromov's models, from which the relations are picked up at random and added to the given group $\Gamma$ (e.g. the free group $\mathbf{F}_{2}$ ).

For a finite subset $A=\left\{C_{1}, \ldots, C_{k}\right\}$ of $\mathscr{C}_{p}$, we set

- $X_{A}:=X-\bigcup_{l=1}^{k} \stackrel{\circ}{C_{l}}$.

- $K_{A}:=\pi_{1}\left(X_{A}\right)$ and $\tilde{X}_{A} \rightarrow X_{A}$ is the corresponding universal cover.

- $\Gamma_{A}$ is the Galois group of the covering map:

$$
\tilde{X}_{A} \rightarrow X_{A} / \Gamma_{p}
$$

The random group is defined by the following.

Definition 4. Fix for every $p \geqslant 1$ a process $\mathbb{P}_{p}$ for selecting random subsets of elements in $\mathscr{C}_{p}$. The random group of order $p$ in the $\left(X, \Gamma,\left\{\Gamma_{p}\right\},\left\{\mathbb{P}_{p}\right\}\right)$-model is the group $\Gamma_{A}$ associated by the construction above to the $\mathbb{P}_{p}$-generic subset $A \subset \mathscr{C}_{p}$. We say that a property $P$ occurs with overwhelming probability in this model if the probability that the random group $\Gamma_{A}$ of order $p$ satisfies $P$ converges to 1 as $p \rightarrow \infty$.

REMARK 5. (1) In all cases considered below, the random process $\left\{\mathbb{P}_{p}\right\}$ is universal in that it does not depend on the deterministic data $\left(X, \Gamma,\left\{\Gamma_{p}\right\}\right)$. More 
precisely, a predetermined process $\mathbb{P}$ is chosen for selecting a random finite subset in an arbitrary finite set, and this process $\mathbb{P}$ is applied recursively to the terms of the sequence $\left(\mathscr{C}_{p}\right)_{p}$.

(2) Most of the results of the present paper extend easily to the case of proper actions on cell complexes with compact quotient. We also note that these models of random group only take into account the 'profinite information' contained in the deterministic data.

We study two special cases of Definition 4: the 'bounded model' and the 'density model'. In the first model, a uniformly bounded number of elements of $\mathscr{C}_{p}$ is chosen at random.

DEFINITION 6 (The bounded model). Fix an integer parameter $c \geqslant 1$. The bounded model over $\left(X, \Gamma,\left\{\Gamma_{p}\right\}\right)$ is the $\left(X, \Gamma,\left\{\Gamma_{p}\right\},\left\{\mathbb{P}_{p}\right\}\right)$-model associated with the process

$\mathbb{P}_{p}:=$ 'choose $c$ chambers in $\mathscr{C}_{p}$, uniformly and independently at random'.

This corresponds to Gromov's 'few-relator model'.

In the second model, the number of chosen chambers in $\mathscr{C}_{p}$ is unbounded and rapidly growing.

Definition 7 (The density model). Fix a real parameter $\delta>0$ (the density). The density model over $\left(X, \Gamma,\left\{\Gamma_{p}\right\}\right)$ is the $\left(X, \Gamma,\left\{\Gamma_{p}\right\},\left\{\mathbb{P}_{p}\right\}\right)$-model associated with the process

$\mathbb{P}_{p}:=$ 'choose $\left|\mathscr{C}_{p}\right|^{\delta}$ chambers in $\mathscr{C}_{p}$, uniformly and independently at random'.

This corresponds to the Gromov 'density model'. As in Gromov models, the bounded model can be seen as a manifestation of the 'density model with $\delta=0$ ' (see also Section 5).

REMARK 8. In a sense, the new models can be thought of as 'mirror images' of the Gromov models: rather than starting with a group with a large supply of quotients, for example nonabelian-free groups, and gradually adding relations at random, we typically start (see below) with lattices in some algebraic group of rank 2, which have 'as many relations as is conceivable' for an infinite group (in particular, they are just infinite up to center), and remove them at random. It is unclear how to randomize (say, residually finite) discrete groups of higher cohomological dimension; for example, the above construction provides a precise meaning for the expression ' $\Gamma$ is a random extension of a lattice in $\operatorname{PSL}_{n}(K)$ ' for $n=3$, where $K$ is a local field-what about $n>3$ ? 


\section{Preliminary results}

Let $\left(X, \Gamma,\left\{\Gamma_{p}\right\}\right)$ be the deterministic data, and let $p \geqslant 1, A \subset \mathscr{C}_{p}, X_{A}, \Gamma_{A}, K_{A}$ (following the notation of Section 2) be fixed throughout the section.

Observe that the sequence of covering spaces $\tilde{X}_{A} \rightarrow X_{A} \rightarrow X_{A} / \Gamma_{p}$ provides an exact sequence (nonsplit in general)

$$
1 \rightarrow K_{A} \rightarrow \Gamma_{A} \rightarrow \Gamma_{p} \rightarrow 1
$$

of discrete groups.

LEMMA 9. If $X$ is contractible, then the following hold.

(1) The homology groups $H_{i}\left(X_{A}, \mathbb{Z}\right)$ vanish for $i \geqslant 2$, and the covering space $\tilde{X}_{A}$ is contractible.

(2) The group $K_{A}$ is a free group on countably many generators.

(3) The group $\Gamma_{A}$ is a finitely presented group of geometric dimension 2. If $|A| \neq$ 0 , then $\Gamma_{A}$ is a strict extension of $\Gamma$.

Proof. The first part of the first assertion is clear, and the second part classically follows from the first $\left(\tilde{X}_{A}\right.$ is weakly homotopy equivalent to a point, and therefore contractible). The universal coefficient theorem

$$
0 \rightarrow \operatorname{Ext}\left(H_{1}\left(X_{A}, \mathbb{Z}\right), \mathbb{Z}\right) \rightarrow H^{2}\left(X_{A}, \mathbb{Z}\right) \rightarrow \operatorname{Hom}\left(H_{2}\left(X_{A}, \mathbb{Z}\right)\right) \rightarrow 0,
$$

where the module $H_{1}\left(X_{A}, \mathbb{Z}\right)$ is free and thus projective, shows that the second integral cohomology $H^{2}\left(X_{A}, \mathbb{Z}\right)$ vanishes. Thus, $K_{A}$ is a free group. This follows from the Stallings-Swan theorem that a group of cohomological dimension 1 is free. (Note that the group $K_{A}$ does not act 'naturally' on a tree in general.) Let us prove (3). Since $\Gamma$ acts freely on $X$ contractible, $X / \Gamma$ is an EilenbergMacLane space, $K(\Gamma, 1)$, and it follows from (1) that $\tilde{X}_{A} / \Gamma_{A}$ is a $K\left(\Gamma_{A}, 1\right)$ and in particular $\Gamma_{A}$ is finitely presented of geometric dimension 2. (If the action $\Gamma \curvearrowright X$ is only assumed to be proper, then the groups $\Gamma_{A}$ are of proper (Bredon) geometric dimension 2.) Assume that $|A| \neq 0$. Let $\gamma$ be the boundary of an element of $A$. If $\Gamma_{A} \rightarrow \Gamma$ is an isomorphism, then $\gamma$ is a boundary in $X_{A}$, and therefore is an equator in a 2-sphere of $X$. This contradicts the fact that $X_{A}$ is aspherical, so $K_{A} \neq 1$, and, by equivariance, $K_{A}$ is infinitely generated.

REMARK 10. If $\Gamma$ is of higher cohomological dimension, then $K_{A}$ is not necessarily free. The density model is interesting to study in this situation, and it seems to be working properly only in 'high density regimes' (this to compensate, 
especially in the case of $\mathrm{SL}_{n}, n \geqslant 4$, for the very strong ambient rigidity properties of the deterministic data).

The following 'flat plane correspondence' assertion is useful in connection with Gromov's periodic flat plane problem.

LEMma 11. Assume that $X$ is a CAT(0) space and that $\Gamma \curvearrowright X$ is isometric. For every flat $\Pi \subset X_{A}$, there exists a flat $\tilde{\Pi} \subset \tilde{X}_{A}$ such that the restriction of $\tilde{\pi}_{A}$ : $\tilde{X}_{A} \rightarrow X_{A}$ to $\tilde{\Pi}$ is an isometry onto $\Pi$. Furthermore, if there exists a subgroup $\Lambda \subset \Gamma, \Lambda \simeq \mathbb{Z}^{2}$, such that $\Pi / \Lambda$ is a compact torus, then there is a corresponding subgroup $\tilde{\Lambda} \subset \Gamma_{A}, \tilde{\Lambda} \simeq \mathbb{Z}^{2}$, such that $\tilde{\Pi} / \tilde{\Lambda}$ is a compact torus, and the following diagram commutes:



Conversely, if $\tilde{\Pi} \subset \tilde{X}_{A}$ is a flat in $\tilde{X}_{A}$, then $\tilde{\pi}_{A}(\tilde{\Pi})$ is a flat in $X_{A}$. If in addition there exists a subgroup $\tilde{\Lambda} \subset \Gamma_{A}, \tilde{\Lambda} \simeq \mathbb{Z}^{2}$, such that $\tilde{\Pi} / \tilde{\Lambda}$ is a compact torus, then its projection $\Lambda$ in $\Gamma$ is a subgroup isomorphic to $\mathbb{Z}^{2}$, such that $\Pi / \Lambda$ is a compact torus.

Proof. Since $\pi_{A}: \tilde{X}_{A} \rightarrow X_{A}$ is a locally isometric covering map and $\mathbb{R}^{2}$ is contractible, the map $j: \mathbb{R}^{2} \rightarrow \Pi \subset X_{A}$ admits a unique isometric lifting $\tilde{j}: \mathbb{R}^{2} \rightarrow \tilde{\Pi} \subset \tilde{X}_{A}$ through any point $\tilde{j}(0)=\tilde{x} \in \tilde{X}_{A}$ such that $\pi_{A}(\tilde{x})=j(0)=: x$, such that the following diagram commutes:

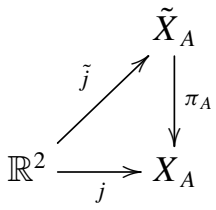

The map $\tilde{\pi}_{A}$, being a local isometry, restricts to an isometry from $\tilde{\Pi}$ onto $\Pi$. Let $\Lambda \subset \Gamma$ be a subgroup isomorphic to $\mathbb{Z}^{2}$ such that $\Pi / \Lambda$ is a compact torus. Let $s \in \Lambda$, and let $g_{s} \in \Gamma_{A}$ be any lift of $s \in \Lambda$. By equivariance of $\pi_{A}$, we have $\pi_{A}\left(g_{s}(\tilde{x})\right)=s x$. Choose $k_{s} \in K_{A}$ such that $k_{s} g_{s} \tilde{x} \in \tilde{\Pi}$. Then $\left(k_{s} g_{s}\right)^{-1}(\tilde{\Pi})$ is an isometric lifting of $\Pi$ which contains $\tilde{x}$. Uniqueness of liftings implies that $k_{s} g_{s}(\tilde{\Pi})=\tilde{\Pi}$. Let $\{a, b\}$ be a generating set of $\Lambda$, and let $\tilde{a}, \tilde{b} \in \Gamma_{A}$ be such that $\tilde{a}(\tilde{\Pi})=\tilde{\Pi}$ and $\tilde{b}(\tilde{\Pi})=\tilde{\Pi}$. Since $\pi_{A}(\tilde{a} \tilde{b} \tilde{x})=a b \pi_{A}(\tilde{x})=b a \pi_{A}(\tilde{x})=\pi_{A}(\tilde{b} \tilde{a} \tilde{x})$ 
and $\pi_{A}: \tilde{\Pi} \rightarrow \Pi$ is isometric, the elements $\tilde{a}$ and $\tilde{b}$ of $\Gamma_{A}$ commute and therefore generate an (infinite torsion-free) abelian group $\tilde{\Lambda} \subset \Gamma_{A}$. If $\Lambda \simeq \mathbb{Z}$, let $\tilde{c}$ be a generator, and let $c \in \Gamma_{p}$ be its image. Then $c(\Pi)=\Pi$ and $\Lambda \subset\langle c\rangle$, contradicting $\Lambda \simeq \mathbb{Z}^{2}$. Therefore $\tilde{\Lambda} \curvearrowright \tilde{\Pi}$ is conjugate to a discrete action of $\mathbb{Z}^{2}$ on $\mathbb{R}^{2}$, and hence $\tilde{\Pi} / \tilde{\Lambda}$ is compact.

Conversely, let $\Pi$ be the image of $\tilde{\Pi}$ under $\pi_{A}$. Since $\pi_{A \mid \tilde{\Pi}}$ is a locally isometric covering map, $\Pi$ is either a torus, a cylinder, or a flat plane. The first two possibilities are incompatible with the fact that $\Pi \subset X_{A} \subset X$, where $X$ is a CAT(0) space of dimension 2. In particular, we see that, if $\tilde{\Lambda}=\langle\tilde{a}, \tilde{b}\rangle$ is a subgroup isomorphic to $\mathbb{Z}^{2}$ acting on $\tilde{\Pi}$ with $\tilde{\Pi} / \tilde{\Lambda}$ compact, then $K_{A} \cap \tilde{\Lambda}=\{e\}$. But this implies that its image $\Lambda$ in $\Gamma$ is isomorphic to $\mathbb{Z}^{2}$, acting freely on $\Pi$ with $\Pi / \Lambda$ compact.

LemMa 12. Assume that $X$ is a CAT(0) space and that $\Gamma$ acts isometrically. Assume that $A \subset B \subset \mathscr{C}_{p}$.

(1) If $\Gamma_{A}$ is Gromov hyperbolic, then $\Gamma_{B}$ is Gromov hyperbolic.

(2) If $\Gamma_{B}$ contains a subgroup isomorphic to $\mathbb{Z}^{2}$, then $\Gamma_{A}$ contains a subgroup isomorphic to $\mathbb{Z}^{2}$.

Proof. (1) By invariance under quasiisometry, it is enough to prove that $\tilde{X}_{B}$ is hyperbolic. If it is not hyperbolic, then, being a CAT(0) space, it contains a flat plane $\Pi \simeq \mathbb{R}^{2} \hookrightarrow \tilde{X}_{B}$ by the flat plane theorem. Since the image of $\Pi$ in $X_{B}$ under the covering map $\pi_{B}: \tilde{X}_{B} \rightarrow X_{B}$ is a flat plane, the space $X_{A}$ contains a flat plane, and therefore $\tilde{X}_{A}$ contains a flat plane by Lemma 11 . Therefore, $\tilde{X}_{A}$ is not hyperbolic and $\Gamma_{A}$ is not a hyperbolic group, contrary to assumption. Thus $\Gamma_{B}$ is hyperbolic.

(2) Since $A \subset B$, the map $\tilde{X}_{B} \rightarrow X_{B} \hookrightarrow X_{A}$ lifts to a map

$$
\tilde{X}_{B} \rightarrow \tilde{X}_{A} \rightarrow X_{A} .
$$

Let $Y=\tilde{X}_{A}, \Delta=\Gamma_{A}$, and let $C$ be the pull-back of $B \backslash A \subset X$ in $Y$. Let also $\tilde{\Lambda} \subset \Gamma_{B}$ be a subgroup isomorphic to $\mathbb{Z}^{2}$, and let $\tilde{\Pi} \subset \tilde{X}_{B}$ be the corresponding periodic flat. We apply Lemma 11 to the isometric action $\Delta \curvearrowright Y$, and the subset $C$ of $Y$. Then $Y_{C}$ coincides with the image of $\tilde{X}_{B}$ in $Y$ and $\tilde{Y}_{C}=\tilde{X}_{B}, \Delta_{C}=\Gamma_{B}$. It follows that $\Pi=\tilde{\pi}_{C}(\tilde{\Pi})$ is a flat in $Y_{C} \subset Y=\tilde{X}_{A}$ and that the projection $\Lambda$ of $\tilde{\Lambda}$ in $\Delta$ is a subgroup isomorphic to $\mathbb{Z}^{2}$, such that $\Pi / \Lambda$ is compact. This shows that $\Gamma_{A}$ contains a copy of $\mathbb{Z}^{2}$.

The same is true for the isolated flats property. 
LEMMA 13. Assume that $X$ is a $C A T(0)$ space and that $\Gamma$ acts isometrically. If $X$ has the isolated flats property, then $\tilde{X}_{A}$ has the isolated flats property.

\section{Random periodic flat plane problems I}

We consider the bounded model first.

Recall that a countable group $\Gamma$ is called virtually indicable if some finite index subgroup of $\Gamma$ admits an infinite abelian quotient.

The main theorem in this section is a result that is significantly more general than Theorem 1 but is restricted to the bounded model. It produces groups which are infinitesimal perturbations of the deterministic data.

Lemma 14. Assume that the deterministic data $\left(X, \Gamma,\left\{\Gamma_{p}\right\}\right)$ satisfies:

- $X$ is a $C A T(0)$ space and $\Gamma$ acts isometrically;

- $\Gamma_{p} \triangleleft \Gamma$, and $\left[\Gamma: \Gamma_{p}\right] \rightarrow_{p} \infty$;

- $\Gamma$ is not virtually indicable;

- $\mathbb{Z}^{2} \hookrightarrow \Gamma$;

then the random group in the bounded (X, $\left.\Gamma,\left\{\Gamma_{p}\right\}\right)$-model contains a copy of $\mathbb{Z}^{2}$ with overwhelming probability.

Proof. Let $\Lambda$ be a subgroup of $\Gamma$ isomorphic to $\mathbb{Z}^{2}$. Let $\Pi \hookrightarrow X$ be a flat plane on which $\Lambda$ acts freely with $X / \Lambda$ a compact torus (which exists by the flat torus theorem). Consider the subgroup

$$
\Lambda_{p}=\Gamma_{p} \cap \Lambda,
$$

and let $F_{p}$ be the set of $\Lambda_{p}$-orbits of chambers in $\Pi$. Since

$$
\left[\Lambda: \Lambda_{p}\right] \leqslant\left[\Gamma: \Gamma_{p}\right],
$$

the set $F_{p}$ is finite. We denote by $F_{p}^{\prime} \subset \mathscr{C}_{p}$ the image of $F_{p}$ into $\mathscr{C}_{p}$ under the map which associates to a $\Lambda_{p}$-orbit in $\Pi$ its corresponding $\Gamma_{p}$-orbit in $X$.

Let $\left(\Gamma_{A_{p}}, \tilde{X}_{A_{p}}\right)$ be the random group associated with a random subset $A_{p} \subset \mathscr{C}_{p}$ in the bounded model (so $\left|A_{p}\right|=c \geqslant 1$ is a fixed integer). We will show that

$$
\mathbb{P}\left(A_{p} \cap \Pi \neq \emptyset\right) \underset{p \rightarrow \infty}{\longrightarrow} 0 .
$$

Assume toward a contradiction that the limit is not zero. Then there exist $\delta>0$ and a sequence $p_{1}<p_{2}<p_{3}<\cdots$ of integers such that, for all $i \geqslant 1$, the random 
set $A_{p_{i}}$ in $\mathscr{C}_{p_{i}}$ contains, with probability at least $\delta>0$, an equivariant chamber $C$ such that $C \cap \Pi \neq \emptyset$ :

$$
\mathbb{P}\left(\exists C \in A_{p_{i}} \mid C \cap \Pi \neq \emptyset\right) \geqslant \delta .
$$

A fortiori,

$$
\sum_{C \in A_{p_{i}}} \mathbb{P}(C \cap \Pi \neq \emptyset) \geqslant \delta .
$$

Thus, for all $i \geqslant 1$,

$$
\left|\left\{C \in \mathscr{C}_{p_{i}} \mid C \cap \Pi \neq \emptyset\right\}\right| \geqslant \frac{\delta}{c}\left|\mathscr{C}_{p_{i}}\right| .
$$

Since the condition $C \cap \Pi \neq \emptyset$ is equivalent to the fact that $C \in F_{p_{i}}^{\prime}$, we obtain

$$
\left|F_{p_{i}}\right| \geqslant\left|F_{p_{i}}^{\prime}\right| \geqslant\left|\left\{C \in \mathscr{C}_{p_{i}} \mid C \cap \Pi \neq \emptyset\right\}\right| \geqslant \frac{\delta}{c}\left|\mathscr{C}_{p_{i}}\right|
$$

Consider the groups $G_{p}=\Gamma / \Gamma_{p}$ and $H_{p}=\Lambda / \Lambda_{p}$, which act freely on $\mathscr{C}_{p}$ and $F_{p}$, respectively.

It follows that there exists a constant $\kappa$, which depends only on $\delta, c$, the number of $\Gamma$-orbit of chambers in $X$, and the number of $\Lambda$-orbits of chambers in $\Pi$, such that

$$
\left[G_{p_{i}}: H_{p_{i}}\right] \leqslant \kappa .
$$

Hence, for every $i$, the subgroup $\Lambda \Gamma_{p_{i}}$ of $\Gamma$ is (by the isomorphism theorems) of index

$$
\left[\Gamma: \Lambda \Gamma_{p_{i}}\right] \leqslant \kappa
$$

As $\Gamma$ is finitely generated (for it acts freely on $X$ with $X / \Gamma$ compact), the family $\mathcal{F}$ of subgroups of index at most $\kappa$ is finite.

A finitely generated group $\Gamma$ is not virtually indicable if and only if every finite index subgroup $\Gamma_{0}$ of $\Gamma$ has finite abelianization $\Gamma_{0} /\left[\Gamma_{0}, \Gamma_{0}\right]$. Since $\Lambda \Gamma_{p_{i}} \in \mathcal{F}$, we have

$$
\left|\Lambda \Gamma_{p_{i}} /\left[\Lambda \Gamma_{p_{i}}, \Lambda \Gamma_{p_{i}}\right]\right|<\gamma
$$

for some fixed $\gamma>0$, not depending on $i$. On the other hand, since $\Lambda \simeq \mathbb{Z}^{2}$,

$$
H_{p_{i}} \subset \Lambda \Gamma_{p_{i}} /\left[\Lambda \Gamma_{p_{i}}, \Lambda \Gamma_{p_{i}}\right]
$$

and as

$$
\left|H_{p_{i}}\right| \underset{\left|G_{p_{i}}\right| \rightarrow \infty}{\longrightarrow} \infty
$$


we have

$$
\left|\Lambda \Gamma_{p_{i}} /\left[\Lambda \Gamma_{p_{i}}, \Lambda \Gamma_{p_{i}}\right]\right| \underset{p_{i} \rightarrow \infty}{\longrightarrow} \infty,
$$

which gives the desired contradiction. Thus,

$$
\mathbb{P}\left(A_{p} \cap \Pi \neq \emptyset\right) \underset{p \rightarrow \infty}{\longrightarrow} 0 .
$$

We now apply Lemma 11, whose assumptions are satisfied with overwhelming probability. This shows that $\mathbb{Z}^{2} \hookrightarrow \Gamma_{A_{p}}$ with overwhelming probability.

Theorem 16 below can be expressed by saying that 'the periodic flat plane problem is stable under randomization in the bounded model', under suitable assumptions. More precisely, we have the following.

DEFINITION 15 . We say that a property $P$ is stable under randomization in the $\left(X, \Gamma,\left\{\Gamma_{p}\right\},\left\{\mathbb{P}_{p}\right\}\right)$-model if the random group has property $P$ with overwhelming probability provided that the deterministic data $\left(X, \Gamma,\left\{\Gamma_{p}\right\}\right)$ has property $P$.

THEOREM 16. Let $\left(X, \Gamma,\left\{\Gamma_{p}\right\}\right)$ be the deterministic data, where $\Gamma$ acts isometrically on $X C A T(0)$, and $\Gamma_{p} \triangleleft \Gamma$ is a sequence of normal subgroups such that $\left[\Gamma: \Gamma_{p}\right] \rightarrow \infty$. If $\Gamma$ is not virtually indicable, then the periodic flat plane alternative is stable under randomization in the bounded $\left(X, \Gamma,\left\{\Gamma_{p}\right\}\right)$ model.

Proof. Assume that $\Gamma$ satisfies the periodic flat plane alternative and let us prove that the random group does. If $\Gamma$ contains $\mathbb{Z}^{2}$, then Lemma 14 shows more: every copy of $\mathbb{Z}^{2}$ eventually survives in the random group. If $\Gamma$ does not contain $\mathbb{Z}^{2}$, then it is hyperbolic, and Lemma 12 shows that the random group is hyperbolic as well.

According to the 'flat closing conjecture' (which is equivalent to the hypothesis that the periodic flat plane problem always has a positive answer), one expects that virtual indicability does not play a role regarding the existence of a periodic flat plane in the bounded model. The conjecture implies that, for arbitrary deterministic data $\left(X, \Gamma,\left\{\Gamma_{p}\right\}\right)$, where $X$ is $\operatorname{CAT}(0)$ and $\Gamma$ acts isometrically, the periodic flat plane alternative is stable under randomization in the bounded $(X$, $\left.\Gamma,\left\{\Gamma_{p}\right\}\right)$-model. This can be checked easily in a few situations, including spaces with isolated flats, using Lemma 13.

Let us conclude this section with a statement which (in the case of the bounded model) weakens the assumption on the deterministic data for the $\mathbb{Z}^{2} \hookrightarrow \Gamma$ assertion in Theorem 1. 
Let $k$ be a nonarchimedean local field with discrete valuation, and let $\mathbf{G}$ be an algebraic group of rank 2 over $k$. Let $X$ be the associated Bruhat-Tits building, let $\Gamma$ be a uniform lattice in $\mathbf{G}$ acting freely on $X$, let $\left\{\Gamma_{p}\right\}_{p}$ be a sequence of finite index normal subgroups, and let us take $\left(X, \Gamma,\left\{\Gamma_{p}\right\}\right)$ as the deterministic data.

COROllary 17. If $\Gamma$ is the random group in the bounded $\left(X, \Gamma,\left\{\Gamma_{p}\right\}\right)$-model, then $\mathbb{Z}^{2} \hookrightarrow \Gamma$ with overwhelming probability.

Note that such deterministic data can be constructed. Indeed, let $\Gamma^{\prime}$ be any uniform lattice in $\mathbf{G}$ (see [19, Ch. IX.3]; if the characteristic of $k$ is zero, then all lattices are all uniform). Then, by a well-known result of Selberg, $\Gamma$ has a torsion-free subgroup of finite index $\Gamma$, and since $\mathbf{G}$ over $k$ is a linear group, Malcev's theorem shows that $\Gamma$ is residually finite. Now $\Gamma_{p}$ may be taken to be any sequence of finite index subgroups of $\Gamma$, for example a sequence of normal subgroups with trivial intersection.

Proof. Being an algebraic group of rank 2, G satisfies Kazhdan's property T (see [5, Ch. 1, Theorem 1.6.1]), and so does the lattice $\Gamma$. A fortiori, $\Gamma$ is not virtually indicable, and therefore Lemma 14 applies.

\section{Critical densities, factors, and lifts}

This section defines two relevant critical densities for studying the periodic flat plane problem in the density model: $\delta_{\mathbb{R}^{2}}$ (the critical density for being hyperbolic) and $\delta_{\mathbb{Z}^{2}}$ (the critical density for containing $\mathbb{Z}^{2}$ ). Existence follows from the 'lift/quotient stability in the density model' of the corresponding group property (compare Proposition 23).

We call a group property $P$ lift stable (respectively, factor stable or quotient stable), if, for every surjective morphism $\Gamma \rightarrow \Gamma^{\prime}$ where $\Gamma^{\prime}$ (respectively, $\Gamma$ ) has property $P$, then $\Gamma$ (respectively, $\Gamma^{\prime}$ ) has property P. It is clear that the following hold.

- $P$ lift stable $\Leftrightarrow \neg P$ factor stable.

- $P$ lift stable and $P$ factor stable implies that $P$ trivial. (In this respect, factorstable and lift-stable properties are 'half-trivial' properties.)

The following result explains the origin, for lift-/factor-stable group properties, of transition thresholds observed in the density models.

Proposition 18. Let $P$ be a lift-stable (respectively, $P^{\prime}$ be a factor-stable) group property and let $\left(\Gamma, X,\left\{\Gamma_{p}\right\}\right)$ be the deterministic data. There exists a 
critical parameter $\delta_{P}=\delta_{P}\left(\Gamma, X,\left\{\Gamma_{p}\right\}\right)\left(\right.$ respectively, $\left.\delta_{P^{\prime}}=\delta_{P^{\prime}}\left(\Gamma, X,\left\{\Gamma_{p}\right\}\right)\right)$ such that the random group $\Gamma$ in the density model of parameter $\delta$ over $\left(\Gamma, X,\left\{\Gamma_{p}\right\}\right)$ satisfies the following:

- $\Gamma$ has property $P$ if $\delta>\delta_{P}$ (respectively, $\Gamma$ has property $P^{\prime}$ if $\delta<\delta_{P^{\prime}}$ );

- $\Gamma$ does not have property $P$ if $\delta<\delta_{P}$ (respectively, $\Gamma$ does not have property $P^{\prime}$ if $\left.\delta>\delta_{P}^{\prime}\right)$;

(with overwhelming probability). In particular, $\delta_{\neg P} \leqslant \delta_{P}$ and $\delta_{P^{\prime}} \leqslant \delta_{\neg P^{\prime}}$ respectively-leading to a 'threshold':



'Half-trivial' properties have one threshold

It seems that in known cases $\delta_{P}=\delta_{\neg P}$. For $P$ such that $\delta_{P}>\delta_{\neg P}$, it could be informative to determine the true behavior of the probabilities on the interval between $\delta_{\neg P}$ and $\delta_{P}$.

EXAMPLE 19. The proposition shows the existence of critical densities for the following.

- Kazhdan's property T (factor stable), say $\delta_{T}$.

- Serre's property FA (factor stable), say $\delta_{F A}$.

- Largeness, which is the existence of a finite index subgroup of $\Gamma$ surjecting to a nonabelian-free group (lift stable), say $\delta_{\rightarrow F_{2}}^{\text {virt }}$.

- Virtual indicability (lift stable), say $\delta_{\rightarrow \mathbb{Z}}^{\text {virt }}$.

Here,

$$
\delta_{T} \leqslant \delta_{F A} \leqslant \delta_{\rightarrow F_{2}}^{\mathrm{virt}} \leqslant \delta_{\rightarrow \mathbb{Z}}^{\mathrm{virt}} .
$$

Observe that, if $P \Rightarrow P^{\prime}$, where both $P, P^{\prime}$ are factor stable/both are lift stable, or if $P \Rightarrow \neg P^{\prime}$, where $P$ is factor stable and $P^{\prime}$ is lift stable, then $\delta_{P} \leqslant \delta_{P^{\prime}}$.

The proof of Proposition 18 will follow from an assertion that 'the random group at density $\delta^{\prime}$ is a quotient of the random group at a higher density $\delta>\delta^{\prime}$, which we make precise in Lemma 21. The existence of transition thresholds does not generalize to properties which are not lift stable or factor stable. For example, a property like $T \vee \stackrel{\text { virt }}{\rightarrow} \mathbb{Z}$ admits a priori two thresholds. In general, the above 
result can be extended to any finite conjunction of properties which are lift stable or factor stable, resulting in a finite number of phase transitions.

We prove Lemma 21 in a more general model, called the ' $\alpha$-model', which extends both the bounded model (where $\alpha$ is bounded) and the density model of parameter $\delta$ for any $\delta>0\left(\right.$ where $\left.\alpha_{p}=\left|\mathscr{C}_{p}\right|^{\delta}\right)$.

Definition 20. Let $\left(X, \Gamma,\left\{\Gamma_{p}\right\}\right)$ be the deterministic data, and let $\alpha: \mathbb{N} \rightarrow \mathbb{N}$ be an increasing sequence. The $\alpha$-model over $\left(X, \Gamma,\left\{\Gamma_{p}\right\}\right)$ is the $\left(X, \Gamma,\left\{\Gamma_{p}\right\}\right.$, $\left\{\mathbb{P}_{p}\right\}$ )-model of random groups in which $\mathbb{P}_{p}$ selects $\alpha_{p}$ chambers in $\mathscr{C}_{p}$, uniformly and independently at random. Given $\alpha, \beta: \mathbb{N} \rightarrow \mathbb{N}$, we say that the $\alpha$-model is dominated by the $\beta$-model if $\alpha_{p} \leqslant \beta_{p}$ for every $n$ sufficiently large.

Zero density models, in which say $\alpha_{p}:=o\left(\left|\mathscr{C}_{p}\right|^{\delta}\right)$ for every $\delta>0$ (this includes the bounded model), can be used to provide variations on the model density $\delta>0$, for example: $\alpha_{p}:=\left|\mathscr{C}_{p}\right|^{\delta}+o\left(\left|\mathscr{C}_{p}\right|^{\delta}\right)$.

The bounded model is dominated by the density model of parameter $\delta$, which is dominated by the density model of parameter $\delta^{\prime}>\delta$. Lemma 21 makes explicit the fact that, if $\alpha$ dominates $\beta$, then 'the random group in the $\alpha$-density model is a quotient of the random group in the $\beta$-density model'. We prove the case of lift-stable properties only, since the corresponding statement for factor-stable properties is analogous.

LEMMA 21. Let $P$ be a lift-stable group property, and let $\left(\Gamma, X,\left\{\Gamma_{p}\right\}\right)$ be the deterministic data. If the $\alpha$-model on $\left(\Gamma, X,\left\{\Gamma_{p}\right\}\right)$ is dominated by the $\beta$-model on $\left(\Gamma, X,\left\{\Gamma_{p}\right\}\right)$, then the probability that the random group has property $P$ in the $\alpha$-model is dominated by the probability that the random group has property $P$ in the $\beta$-model.

In particular, if $P$ occurs with overwhelming probability in the $\alpha$-model, then it does so in the $\beta$-model, establishing Proposition 18.

Proof. If $P$ is lift stable, then

$$
\begin{aligned}
& \mid\left\{c_{1}, \ldots, c_{\alpha_{p}} \in \mathscr{C}_{p} \mid \Gamma_{\left\{c_{1}, \ldots, c_{\left.\alpha_{p}\right\}}\right\}} \text { has property P }\right\} \mid \\
& \quad=\frac{\mid\left\{c_{1}, \ldots, c_{\beta_{p}} \in \mathscr{C}_{p} \mid \Gamma_{\left\{c_{1}, \ldots, c_{\alpha_{p}}\right\}} \text { has property } \mathrm{P}\right\} \mid}{\left|\mathscr{C}_{p}\right|^{\beta_{p}-\alpha_{p}}} \\
& \quad \leqslant \frac{\mid\left\{c_{1}, \ldots, c_{\beta_{p}} \in \mathscr{C}_{p} \mid \Gamma_{\left\{c_{1}, \ldots, c_{\beta_{p}}\right\}} \text { has property } \mathrm{P}\right\} \mid}{\left|\mathscr{C}_{p}\right|^{\beta_{p}-\alpha_{p}}} .
\end{aligned}
$$

Therefore, if $A_{\alpha}$ denotes the random subset of $\mathscr{C}_{p}$ in the $\alpha$-model and $A_{\beta}$ denotes the random subset of $\mathscr{C}_{p}$ in the $\beta$-model, then 


$$
\mathbb{P}\left(\Gamma_{A_{\alpha}} \text { has property } \mathrm{P}\right) \leqslant \mathbb{P}\left(\Gamma_{A_{\beta}} \text { has property } \mathrm{P}\right) .
$$

This proves the assertion.

However, a property like 'hyperbolicity' is neither factor stable nor lift stable, even though Gromov establishes a sharp phase transition in his density model.

The following weakening of lift/factor stability is consistent with the density model and allows for more general properties.

DEFINITION 22. A group property $P$ is said to be lift stable (respectively, factor stable or quotient stable) in the density model if the random group at density $\delta$ satisfies property $P$ with overwhelming probability whenever the random group at density $\delta^{\prime}<\delta$ (respectively, $\delta^{\prime}>\delta$ ) satisfies property $P$ with overwhelming probability.

Thus, we have (tautologically) a critical density $\delta_{P}\left(\Gamma, X,\left\{\Gamma_{p}\right\}\right)$ for every liftstable property $P$ in the density model, and similarly for factor-stable properties. Furthermore,

$$
\delta_{\neg P}\left(\Gamma, X,\left\{\Gamma_{p}\right\}\right) \leqslant \delta_{P}\left(\Gamma, X,\left\{\Gamma_{p}\right\}\right)
$$

if $P$ is lift stable and $\neg P$ factor stable.

Proposition 23. Let $\left(\Gamma, X,\left\{\Gamma_{p}\right\}\right)$ be the deterministic data, where $X$ is a $C A T(0)$ space and $\Gamma$ acts isometrically. Then the following hold.

(1) Gromov hyperbolicity is lift stable in the density model over $\left(\Gamma, X,\left\{\Gamma_{p}\right\}\right)$. Therefore, there exists a critical density, denoted $\delta_{\mathbb{R}^{2}}\left(\Gamma, X,\left\{\Gamma_{p}\right\}\right)$, for the random group in the density model to be hyperbolic.

(2) The existence of subgroups isomorphic to $\mathbb{Z}^{2}$ is factor stable in the density model. Therefore, there exists a critical density, denoted $\delta_{\mathbb{Z}^{2}}\left(\Gamma, X,\left\{\Gamma_{p}\right\}\right)$, for the random group in the density model to contain a subgroup isomorphic to $\mathbb{Z}^{2}$.

By the periodic flat plane theorem,

$$
\delta_{\mathbb{R}^{2}}\left(\Gamma, X,\left\{\Gamma_{p}\right\}\right) \geqslant \delta_{\mathbb{Z}^{2}}\left(\Gamma, X,\left\{\Gamma_{p}\right\}\right) .
$$

Problem 24. Given the deterministic data $\left(\Gamma, X,\left\{\Gamma_{p}\right\}\right)$, where $X$ is a CAT(0) space and $\Gamma$ acts properly uniformly isometrically, is it true that

$$
\delta_{\mathbb{R}^{2}}\left(\Gamma, X,\left\{\Gamma_{p}\right\}\right)=\delta_{\mathbb{Z}^{2}}\left(\Gamma, X,\left\{\Gamma_{p}\right\}\right) ?
$$


The flat closing conjecture implies that the answer is positive.

Proof of Proposition 23. (1) By Lemma 12(a), we have

$$
\begin{aligned}
& \mid\left\{c_{1}, \ldots, c_{\alpha_{p}} \in \mathscr{C}_{p} \mid \Gamma_{\left\{c_{1}, \ldots, c_{\alpha_{p}}\right\}} \text { is hyperbolic }\right\} \mid \\
& \quad=\frac{\mid\left\{c_{1}, \ldots, c_{\beta_{p}} \in \mathscr{C}_{p} \mid \Gamma_{\left\{c_{1}, \ldots, c_{\alpha_{p}}\right\}} \text { is hyperbolic }\right\} \mid}{\left|\mathscr{C}_{p}\right|^{\beta_{p}-\alpha_{p}}} \\
& \quad \leqslant \frac{\mid\left\{c_{1}, \ldots, c_{\beta_{p}} \in \mathscr{C}_{p} \mid \Gamma_{\left\{c_{1}, \ldots, c_{\beta_{p}}\right\}} \text { is hyperbolic }\right\} \mid}{\left|\mathscr{C}_{p}\right|^{\beta_{p}-\alpha_{p}}} .
\end{aligned}
$$

This shows that the random group in the $\beta$-model is hyperbolic provided that the random in the $\alpha$-model is hyperbolic, for any $\beta$ dominating $\alpha$, which applies in particular to the density model.

(2) The same proof applies (using now Lemma 12(b)), with a reverse inequality. Namely, we have

$$
\begin{aligned}
& \mid\left\{c_{1}, \ldots, c_{\alpha_{p}} \in \mathscr{C}_{p} \mid \Gamma_{\left\{c_{1}, \ldots, c_{\alpha_{p}}\right\}} \text { contains } \mathbb{Z}^{2}\right\} \mid \\
& \quad=\frac{\mid\left\{c_{1}, \ldots, c_{\beta_{p}} \in \mathscr{C}_{p} \mid \Gamma_{\left\{c_{1}, \ldots, c_{\alpha_{p}}\right\}} \text { contains } \mathbb{Z}^{2}\right\} \mid}{\left|\mathscr{C}_{p}\right|^{\beta_{p}-\alpha_{p}}} \\
& \geqslant \frac{\mid\left\{c_{1}, \ldots, c_{\beta_{p}} \in \mathscr{C}_{p} \mid \Gamma_{\left\{c_{1}, \ldots, c_{\beta_{p}}\right\}} \text { contains } \mathbb{Z}^{2}\right\} \mid}{\left|\mathscr{C}_{p}\right|^{\beta_{p}-\alpha_{p}}} .
\end{aligned}
$$

This shows that the random group in the $\alpha$-model contains $\mathbb{Z}^{2}$ provided that the random in the $\beta$-model contains $\mathbb{Z}^{2}$, for any $\beta$ dominating $\alpha$.

REMARK 25. The above discussion of 'lift-stable' and 'factor-stable' properties also applies to the classical Gromov models and shows the existence of critical densities, for example in the density model. Recall that there exist morphisms between the random group of different models at the same density, for example when comparing the usual model with the triangular model; see [21, I.3.g]. The above results are of a different nature in that they concern morphisms between random groups at distinct density regimes in a given model.

\section{General facts on the random group at positive density}

The random group $\Gamma$ in Gromov's density model exhibits the following wellknown phase transition at density $\delta=\frac{1}{2}$ (see [15] and Theorem 11 in [21]).

(1) If $\delta<1 / 2$, then with overwhelming probability $\Gamma$ is infinite hyperbolic, torsion free and of geometric dimension 2 . 
(2) If $\delta>1 / 2$, then with overwhelming probability $\Gamma$ is trivial (either $\{e\}$ or $\mathbb{Z} / 2 \mathbb{Z})$.

In other words, $\delta_{\text {Hyp }}=\delta_{\text {triv }}=\delta_{\neg \text { Hyp }}=\delta_{\neg \text { triv }}=1 / 2$.

The analogous result in our setting takes the following form. The proof, as in Gromov's case, is an illustration of the box principle.

Proposition 26. Let $\left(\Gamma, X,\left\{\Gamma_{p}\right\}\right)$ be the deterministic data, and let $r \in \mathbb{N}_{\geqslant 2}$ be fixed. Then the random space $X_{A}$ in the density model over $\left(X, \Gamma,\left\{\Gamma_{p}\right\}\right)$ exhibits a phase transition with overwhelming probability:

(1) If $\delta<1 / 2$, then the random set $A \subset X$ is $r$-separated (that is, the simplicial distance between any two elements of $A$ is at least $r)$.

(2) If $\delta>1 / 2$, then there exist at least $r$ pairs of adjacent elements of $A$.

Proof. (1) Let $Y_{1}, Y_{2}, Y_{3}, \ldots$ be a sequence of independent uniformly distributed random variables with values in $\mathscr{C}_{p}$, and for $m \leqslant i \leqslant\left|\mathscr{C}_{p}\right|$ denote by $E_{p}^{(i)}(m)$ the event

$$
E_{p}^{(i)}(m)=\left\{\operatorname{diam}\left(Y_{k_{1}}, \ldots, Y_{k_{m}}\right) \geqslant r \mid \forall 1 \leqslant k_{1}<\cdots<k_{m} \leqslant i\right\} .
$$

We have

$$
\begin{aligned}
& \mathbb{P}\left(E_{p}^{(i)}(m)\right) \\
& =\sum_{\left(c_{1}, \ldots, c_{i-1}\right) \in \mathscr{C}_{p}^{i-1}} \mathbb{P}\left(E_{p}^{(i)}(m) \mid\left\{Y_{1}=c_{1}, \ldots, Y_{i-1}=c_{i-1}\right\} \cap E_{p}^{(i-1)}(m)\right) \\
& \quad \cdot \mathbb{P}\left(\left\{Y_{1}=c_{1}, \ldots, Y_{i-1}=c_{i-1}\right\} \cap E_{p}^{(i-1)}(m)\right) .
\end{aligned}
$$

Given the event $\left\{Y_{1}=c_{1}, \ldots, Y_{i-1}=c_{i-1}\right\} \cap E_{p}^{(i-1)}(m)$, namely, that the faces $c_{1}$, $\ldots, c_{i-1}$ have been chosen and any $m$ of them have diameter at least $r$, we obtain, letting $N_{p}\left(c_{1}, \ldots, c_{i-1}\right)$ be the number of faces in $X$ whose union with $m-1$ of the faces $c_{1}, \ldots, c_{i-1}$ forms a set of diameter at most $r$,

$$
\mathbb{P}\left(E_{p}^{(i)}(m) \mid\left\{Y_{1}=c_{1}, \ldots, Y_{i-1}=c_{i-1}\right\} \cap E_{p}^{(i-1)}(m)\right)=1-\frac{N_{p}\left(c_{1}, \ldots, c_{i-1}\right)}{\left|\mathscr{C}_{p}\right|} .
$$

Let $N_{p}$ be the number of faces in the $r$ neighborhood of a single chamber of $X$ (this does not depend on the given chamber). Then

$$
\begin{aligned}
N_{p}\left(c_{1}, \ldots, c_{i-1}\right) & \leqslant N_{p} \cdot\left|\left\{\left(k_{1}, \ldots, k_{m-1}\right) \mid 1 \leqslant k_{1}<\cdots<k_{m-1} \leqslant i-1\right\}\right| \\
& \leqslant N_{p} \cdot(i-1)^{m-1},
\end{aligned}
$$




$$
\begin{aligned}
\mathbb{P}\left(E_{p}^{(i)}(m)\right) \geqslant & \sum_{\left(c_{1}, \ldots, c_{i-1}\right) \in \mathscr{C}_{p}^{i-1}}\left(1-\frac{N_{p} \cdot(i-1)^{m-1}}{\left|\mathscr{C}_{p}\right|}\right) \\
\cdot & \mathbb{P}\left(\left\{Y_{1}=c_{1}, \ldots, Y_{i-1}=c_{i-1}\right\} \cap E_{p}^{(i-1)}(m)\right) \\
= & \left(1-\frac{N_{p} \cdot(i-1)^{m-1}}{\left|\mathscr{C}_{p}\right|}\right) \mathbb{P}\left(E_{p}^{(i-1)}(m)\right) .
\end{aligned}
$$

Using $e^{-2 x} \leqslant 1-x$ for all $x<0.79$, we get

$$
\mathbb{P}\left(E_{n}^{(i)}\right) \geqslant e^{-2 \sum_{j=1}^{i}\left(N_{p} \cdot(j-1)^{m-1} /\left|\mathscr{C}_{p}\right|\right)} \geqslant e^{-2 N_{p}\left(i(i-1) /\left|\mathscr{C}_{p}\right|\right)}
$$

Therefore, if $i=\left|\mathscr{C}_{p}\right|^{\delta}$, where $\delta<1 / 2$, then $\mathbb{P}\left(E_{n}\right)$ is arbitrarily close to 1 . This concludes the proof of (1). The proof of (2) is of a similar nature.

Then the density model exhibits several extra phase transitions as the parameter $\delta$ increases. The transitions depend on the local structure of the initial space $X$ :

Proposition 27. Let $\left(\Gamma, X,\left\{\Gamma_{p}\right\}\right)$ be the deterministic data, and let $r \in \mathbb{N}_{\geqslant 2}$, $k, \ell \in \mathbb{N}_{\geqslant 1}$ be fixed. Then the random space $X_{A}$ in the density model over $(X, \Gamma$, $\left.\left\{\Gamma_{p}\right\}\right)$ admits a phase transition at density $\delta=k /(k+1)$. Namely, the following hold with overwhelming probability.

(1) If $\delta<k /(k+1)$, every ball $B$ of radius $r$ in $X^{2}$ contains at most $k$ elements of $A$.

(2) If $\delta>(k-1) / k$, there exists an $r$-separated set $E \subset X^{(1)}$ of cardinality at least $\ell$, such that every edge $e \in E$ is included in exactly $\min \left(k, q_{e}+1\right)$ elements of $A$.

Proof. (1) Let $Y_{1}, Y_{2}, Y_{3}, \ldots$ be a sequence of independent uniformly distributed random variables with values in $\mathscr{C}_{p}$. For a ball $B \subset X^{(2)}$ of radius $r$, we write $Y_{i} \sim_{B} Y_{j}$ to mean that both $Y_{i}$ and $Y_{j}$ are included in $B$.

Let $B \subset X^{(2)}$ be a ball of radius $r$, and fix $1 \leqslant m_{1}<\cdots<m_{k} \leqslant i$. Since

$$
\mathbb{P}\left(Y_{m_{1}} \sim_{B} Y_{m_{2}} \sim_{B} \cdots \sim_{B} Y_{m_{k}}\right) \leqslant\left(\frac{b^{*}}{\left|\mathscr{C}_{p}\right|}\right)^{k},
$$

where $b^{*}:=\max _{B \subset X^{(2)}}\left|B_{r}\right|$ (which is finite as $X / \Gamma$ is compact), we obtain

$$
\begin{aligned}
\mathbb{P}(\exists 1 & \left.\leqslant m_{1}<\cdots<m_{k} \leqslant i, \exists B, Y_{m_{1}} \sim_{B} Y_{m_{2}} \sim_{B} \cdots \sim_{B} Y_{m_{k}}\right) \\
& \leqslant \sum_{1 \leqslant m_{1}<\cdots<m_{k} \leqslant i} \sum_{f \in \mathscr{C}_{p}} \mathbb{P}\left(Y_{m_{1}} \sim_{B_{f}} Y_{m_{2}} \sim_{B_{f}} \cdots \sim_{B_{f}} Y_{m_{k}}\right)
\end{aligned}
$$




$$
\begin{aligned}
& \leqslant\left|\left\{\left(m_{1}, \ldots, m_{k}\right) \mid 1 \leqslant m_{1}<\cdots<m_{k} \leqslant i\right\}\right| \cdot\left|\mathscr{C}_{p}\right| \cdot\left(\frac{b^{*}}{\left|\mathscr{C}_{p}\right|}\right)^{k} \\
& \leqslant O\left(\frac{i^{k}}{\left|\mathscr{C}_{p}\right|^{k-1}}\right) .
\end{aligned}
$$

Therefore, if $i=\left|\mathscr{C}_{p}\right|^{\delta}$, where $\delta<k /(k-1)$, then with overwhelming probability every ball in $X^{(2)}$ of radius at most $r$ contains at most $k$ elements of the random subset $A$ of $\mathscr{C}_{p}$, at density $\delta$.

(2) Let $Y_{1}, Y_{2}, Y_{3}, \ldots$ be a sequence of independent uniformly distributed random variables with values in $\mathscr{C}_{p}$. Let $\mathscr{F}_{p, k}$ be the set of all $k$-tuples of elements of $\mathscr{C}_{p}$ whose faces are mutually adjacent to a same edge. For every $\psi \in \mathscr{F}_{p, k}$, we write $E_{i, k}(\psi)$ for the event

$E_{i, k}(\psi)=\left\{\exists 1 \leqslant m_{1}, \ldots, m_{k} \leqslant i\left(\forall r \neq s, m_{r} \neq m_{s}\right) \mid Y_{m_{1}}=\psi_{1}, \ldots, Y_{m_{k}}=\psi_{k}\right\}$,

and let $\chi_{i, k}(\psi)$ be the characteristic function of $E_{i, k}(\psi)$. We will estimate the expectation of the random variable

$$
Z_{p, k}:=\sum_{\psi \in \mathscr{F}_{p, k}} \chi_{\left|\mathscr{C}_{p}\right|^{\delta}, k}(\psi)
$$

whenever $\delta \in[(k-1) / k, k /(k+1)]$. Fix $k$ and $\psi \in \mathscr{F}_{p, k}$. Write

$$
\mathbb{P}\left(E_{i, k}(\psi)\right)=\mathbb{P}\left(\bigcup_{m_{1}, \ldots, m_{k}=1 \mid \forall r \neq s, m_{r} \neq m_{s}}^{i} Y_{m_{1}}=\psi_{1}, \ldots, Y_{m_{k}}=\psi_{k}\right) .
$$

We have

$$
\begin{aligned}
\mathbb{P}\left(E_{i, k}(\psi)\right) \geqslant & \sum_{m_{1}, \ldots, m_{k}=1 \mid \forall r \neq s, m_{r} \neq m_{s}}^{i} \mathbb{P}\left(Y_{m_{1}}=\psi_{1}, \ldots, Y_{m_{k}}=\psi_{k}\right) \\
& \left.-\sum_{\substack{m_{1}, \ldots, m_{k}=1 \mid \forall r \neq s, m_{r} \neq m_{s} \\
\mathbb{P}\left(Y_{m_{1}}=\psi_{1}^{\prime}, \ldots, m_{k}^{\prime}=1 \mid \forall r \neq s, m_{r}^{\prime} \neq m_{s}^{\prime}\right.}}^{i} \sum_{m_{k}}^{i}=\psi_{k}, Y_{m_{1}^{\prime}}=\psi_{1}, \ldots, Y_{m_{k}^{\prime}}=\psi_{k}\right)
\end{aligned}
$$

by inclusion-exclusion, where

$$
\mathbb{P}\left(Y_{m_{1}}=\psi_{1}, \ldots, Y_{m_{k}}=\psi_{k}\right)=\frac{1}{\left|\mathscr{C}_{p}\right|^{k}}
$$

and

$$
\mathbb{P}\left(Y_{m_{1}}=\psi_{1}, \ldots, Y_{m_{k}}=\psi_{k}, Y_{m_{1}^{\prime}}=\psi_{1}, \ldots, Y_{m_{k}^{\prime}}=\psi_{k}\right) \leqslant \frac{1}{\left|\mathscr{C}_{p}\right|^{2 k}}
$$


Therefore,

$$
\mathbb{P}\left(E_{i, k}(\psi)\right) \geqslant \frac{i(i-1) \cdots(i-k-1)}{\left|\mathscr{C}_{p}\right|^{k}}-\frac{(i(i-1) \cdots(i-k-1))^{2}}{\left|\mathscr{C}_{p}\right|^{2 k}} .
$$

Substituting $\left|\mathscr{C}_{p}\right|^{\delta}$ for $i$, we deduce

$$
\begin{aligned}
\mathbb{E}\left(Z_{p, k}\right)= & \sum_{\psi \in \mathscr{F}_{p, k}} \mathbb{E}\left(\chi_{\left|\mathscr{C}_{p}\right|^{\delta}, k}(\psi)\right) \\
\geqslant & \left|\mathscr{F}_{p, k}\right|\left(\frac{\left|\mathscr{C}_{p}\right|^{\delta}\left(\left|\mathscr{C}_{p}\right|^{\delta}-1\right) \cdots\left(\left|\mathscr{C}_{p}\right|^{\delta}-k-1\right)}{\left|\mathscr{C}_{p}\right|^{k}}\right. \\
& \left.-\frac{\left|\mathscr{C}_{p}\right|^{2 d}\left(\left|\mathscr{C}_{p}\right|^{\delta}-1\right)^{2} \cdots\left(\left|\mathscr{C}_{p}\right|^{\delta}-k-1\right)^{2}}{\left|\mathscr{C}_{p}\right|^{2 k}}\right) .
\end{aligned}
$$

Since, for $p$ large enough,

$$
\frac{\left|\mathscr{C}_{p}\right|^{\delta}-j}{\left|\mathscr{C}_{p}\right|}<\frac{1}{2}
$$

whenever $\delta<1$ and $j \leqslant k+1$, we have

$$
\frac{\left|\mathscr{C}_{p}\right|^{2 d}\left(\left|\mathscr{C}_{p}\right|^{\delta}-1\right)^{2} \cdots\left(\left|\mathscr{C}_{p}\right|^{\delta}-k-1\right)^{2}}{\left|\mathscr{C}_{p}\right|^{2 k}}<\frac{\left|\mathscr{C}_{p}\right|^{\delta}\left(\left|\mathscr{C}_{p}\right|^{\delta}-1\right) \cdots\left(\left|\mathscr{C}_{p}\right|^{\delta}-k-1\right)}{2^{k}\left|\mathscr{C}_{p}\right|^{k}},
$$

and therefore

$$
\begin{aligned}
\mathbb{E}\left(Z_{p, k}\right) & \geqslant\left|\mathscr{F}_{p, k}\right|\left(1-\frac{1}{2^{k}}\right)\left(\frac{\left|\mathscr{C}_{p}\right|^{\delta}\left(\left|\mathscr{C}_{p}\right|^{\delta}-1\right) \cdots\left(\left|\mathscr{C}_{p}\right|^{\delta}-k-1\right)}{\left|\mathscr{C}_{p}\right|^{k}}\right) \\
& \geqslant\left|\mathscr{F}_{p, k}\right|\left(1-\frac{1}{2^{k}}\right)\left(\frac{\left(\left|\mathscr{C}_{p}\right|^{\delta}-k-1\right)^{k}}{\left|\mathscr{C}_{p}\right|^{k}}\right) .
\end{aligned}
$$

Now,

$$
\left|\mathscr{F}_{p, k}\right| \geqslant \frac{3\left|\mathscr{C}_{p}\right|}{\left(q^{*}+1\right)}
$$

so

$$
\mathbb{E}\left(Z_{p, k}\right) \geqslant \frac{3}{\left(q^{*}+1\right)}\left(1-\frac{1}{2^{k}}\right)\left(\frac{\left(\left|\mathscr{C}_{p}\right|^{\delta}-k-1\right)^{k}}{\left|\mathscr{C}_{p}\right|^{k-1}}\right) .
$$

This shows that

$$
\mathbb{E}\left(Z_{p, k}\right) \rightarrow \infty
$$

whenever $\delta>(k-1) / k$. Since is $X$ uniformly locally bounded, this shows that we can find at least $\ell$ edges $e_{1}, \ldots, e_{\ell}$ in $X$ (for any $\ell$ fixed in advance), having 
at least $k$ chambers adjacent to $e_{j}$ removed. In addition, since the number of such edges grows to infinity, we may suppose that the pairwise distance between these edges is at least $r$ from each other in $X$ (for any $r$ fixed in advance), and since the chambers are chosen independently at random, we may also suppose that exactly $\min \left(k, q_{e_{j}}+1\right)$ distinct chambers will be removed on $e_{1}, \ldots, e_{\ell}$.

Proposition 28. Let $\left(\Gamma, X,\left\{\Gamma_{p}\right\}\right)$ be the deterministic data, and let $\ell \in \mathbb{N}_{\geqslant 2}$. Then the random group $\Gamma_{A}$ in the density model over $\left(X, \Gamma,\left\{\Gamma_{p}\right\}\right)$ satisfies the following with overwhelming probability.

(1) If $\delta<q_{*}(X) /\left(q_{*}(X)+1\right)$, then $\Gamma_{A}$ acts freely on a simply connected space $\tilde{X}_{A}$ without boundary with $X / \Gamma_{A}$ compact.

(2) If $\delta>q_{*}(X) /\left(q_{*}(X)+1\right)$, then $\Gamma_{A}$ splits off a free factor isomorphic to a free group $F_{\ell}$ on $\ell$ generators:

$$
\Gamma_{A} \simeq \Delta * F_{\ell},
$$

where $\Delta$ is a finitely presented group, and $q_{*}(X):=\min _{e \in X^{(1)}} q_{e}$.

In particular,

$$
\delta_{F A}\left(\left(\Gamma, X,\left\{\Gamma_{p}\right\}\right)\right) \leqslant \frac{q_{*}(X)}{q_{*}(X)+1}<1,
$$

where $\delta_{F A}$ is the critical density for Serre's property FA (existence of fixed point for actions on trees).

Proof. The first assertion follows directly from Lemma 27, so we prove (2). Since $X / \Gamma$ is compact, there exists a constant $\gamma>0$ such that, if $q_{*}(X)$ denotes the lowest order of an edge in $X$, then

$$
E_{p}:=\left\{e \in \mathscr{C}_{p}^{(1)} \mid q_{e}=q_{*}(X)\right\}
$$

satisfies

$$
\left|E_{p}\right| \geqslant \gamma\left|\mathscr{C}_{p}^{(1)}\right|
$$

for every $p>0$. Let $k=q_{*}(X)$, and let $\tilde{\mathscr{F}}_{p, k}$ be the set of all $k$-tuples of elements of $\mathscr{C}_{p}$ whose faces are mutually adjacent to a same edge $e \in E_{p}$. The proof of Lemma 27, with the same notation except for $\tilde{\mathscr{F}}_{p, k}$ replacing $\mathscr{F}_{p, k}$, shows that

$$
\mathbb{E}\left(Z_{p, k}\right) \geqslant\left|\tilde{\mathscr{F}}_{p, k}\right|\left(1-\frac{1}{2^{k}}\right)\left(\frac{\left(\left|\mathscr{C}_{p}\right|^{\delta}-k-1\right)^{k}}{\left|\mathscr{C}_{p}\right|^{k}}\right),
$$


and since now

$$
\left|\tilde{\mathscr{F}}_{p, k}\right| \geqslant \frac{3 \gamma\left|\mathscr{C}_{p}\right|}{\left(q^{*}+1\right)},
$$

we obtain $\mathbb{E}\left(Z_{p, k}\right) \rightarrow \infty$. Therefore there exists, with overwhelming probability, an $r$-separated set $E \subset E_{p}$ of cardinal $\ell$, such that every edge $e \in E$ is included in exactly $q_{*}(X)+1$ elements of $A \subset \mathscr{C}_{p}$. Let $V_{p}$ be the quotient space $X / \Gamma_{p}$, let $T_{p}$ be a maximal tree of the 1-skeleton of $V_{p}$, and choose a root $s_{p} \in T_{p}$. Let $V_{p}^{\prime}$ be the topological space obtained from $V_{p}$ by collapsing $T_{p}$ to $s_{p}$, which is homotopy equivalent to $V_{p}$. The elements of $E / \Gamma$, being face-free edges in

$$
V_{A}:=X_{A} / \Gamma_{p} \subset V_{p},
$$

form a wedge sum $B_{\ell}$ of $\ell$ edges in the collapse $V_{A}^{\prime}$ of $V_{A}$ in $V_{p}^{\prime}$, and $V_{A}^{\prime}$ is the wedge sum of $B_{\ell}$ and a compact complex $V_{A}^{\prime \prime}$. The van Kampen theorem shows that $\Gamma_{A} \simeq \pi_{1}\left(V_{A}^{\prime}\right)$ splits as

$$
\pi_{1}\left(V_{A}^{\prime}\right)=\pi_{1}\left(V_{A}^{\prime \prime}\right) * \pi_{1}\left(B_{\ell}\right),
$$

where $\pi_{1}\left(B_{\ell}\right) \simeq F_{\ell}$ and $\pi_{1}\left(V_{A}^{\prime \prime}\right)$ is finitely presented.

In particular, we have

$$
\delta_{\neg F A}\left(\left(\Gamma, X,\left\{\Gamma_{p}\right\}\right)\right) \leqslant \frac{q_{*}(X)}{q_{*}(X)+1},
$$

proving the proposition.

\section{Random periodic flat plane problems II}

We now prove the implication

$$
\delta<\frac{5}{8} \Rightarrow \mathbb{Z}^{2} \hookrightarrow \Gamma
$$

in Theorem 1, and similar assertions over local rings.

In Theorem 1, the fixed global field (denoted $k$ ) is $\mathbf{F}_{q}(y)$, and the uniform lattice $\Gamma$ in the deterministic data $\left(X, \Gamma,\left\{\Gamma_{p}\right\}\right)$ is the Cartwright-Steger lattice [7] which is of type $\tilde{A}_{2}$ over $\mathbf{F}_{q}((y))$. The presentation of $\Gamma$ by Lubotzky, Samuels, and Vishne in [18] is especially useful, where the congruence subgroups $\left\{\Gamma_{p}\right\}$ are described explicitly to illustrate the Ramanujan property of their quotients. We first review briefly the construction for notational purposes, referring to [18] for complete details.

Let $D$ be the central simple algebra of degree 3 over $k$ defined by

$$
D=\bigoplus_{i, j=0}^{2} k \xi_{i} z^{j}
$$


with relations $z \xi_{i}=\phi\left(\xi_{i}\right) z$ and $z^{3}=1+y$, where $\phi$ is a generator of $\operatorname{Gal}\left(\mathbf{F}_{q^{3}} / \mathbf{F}_{q}\right)$ and $\xi_{i}=\phi^{i}\left(\xi_{0}\right)$ is a basis for $\mathbf{F}_{q^{3}}$ over $\mathbf{F}_{q}$. Associated with $D$ are algebraic groups over $k$ defined by $\tilde{\mathbf{G}}=D^{\times}$and $\mathbf{G}=D^{\times} / k^{\times}$. For a valuation $v$ on $k$, we let $D_{v}=D \otimes_{k} k_{v}$, and we say that $D_{v}$ splits whenever $D_{v} \simeq \mathrm{M}_{3}\left(k_{v}\right)$.

Let $T=\left\{v_{1 / y}, v_{1+y}\right\}$ consisting of the degree valuation $v_{1 / y}$ on $k$, and the valuation $v_{1+y}$ associated with the prime $1+y$, namely, $v_{1+y}\left((1+y)^{i} f / g\right)=i$ where the polynomials $f, g$ are prime to $(1+y)$. Then, by [18, Proposition 3.1], the algebra $D_{v}$ splits for all valuations $v \notin T$ on $k$, while it is a division algebra for $v \in T$.

Let $v_{0}=v_{y} \notin T$ and $R_{0}=\left\{x \in k \mid v(x) \geqslant 0, \forall v \neq v_{0}\right\}=\mathbf{F}_{q}[1 / y]$. Since $D_{v_{0}}$ splits, we have $\mathbf{G}\left(k_{v_{0}}\right) \simeq \mathrm{PGL}_{3}\left(\mathbf{F}_{q}((y))\right)$, so $\mathbf{G}\left(R_{0}\right)$ embeds as a discrete subgroup of $\mathrm{PGL}_{3}\left(\mathbf{F}_{q}((y))\right)$. Since $T \neq \emptyset$ and $\mathbf{G}\left(k_{v}\right)$ is compact for $v \in T$, then Strong Approximation (see [18, Section 4]) shows that $\mathbf{G}\left(R_{0}\right)$ is a cocompact lattice in $\operatorname{PGL}_{3}\left(\mathbf{F}_{q}((y))\right)$.

The Cartwright-Steger lattice [7, Section 2] is the subgroup $\Gamma$ of $\mathbf{G}\left(R_{0}\right)$ defined as follows. We note that, as stated above, the lattice $\mathbf{G}\left(R_{0}\right)$ is well defined only up to commensurability. A strict definition of $\mathbf{G}\left(R_{0}\right)$ depends upon fixing a embedding $\mathbf{G}$ into a linear group over $k$, which is chosen here to be $\mathrm{GL}_{9}(k)$ (see [18, Proposition 3.3]), so that $\mathbf{G}\left(R_{0}\right):=\mathbf{G}(k) \cap \mathrm{M}_{9}\left(R_{0}\right)$. Then $\Gamma$ consists of matrices of $\mathbf{G}\left(R_{0}\right)$ whose reductions modulo $1 / y$ are upper triangular with $3 \times 3$ identity blocks on the diagonal.

Another description of $\Gamma$ from [18, Section 4] is as follows. Let $R$ be the subring of $k$ given by $R=\mathbf{F}_{q}[y, 1 / y, 1 /(1+y)]$, and let $A(R)$ be the $R$-algebra $A(R)=\bigoplus_{i, j=0}^{2} R \xi_{i} z^{j}$ having the same defining relations as $D$, so that $D$ appears as the algebra of central fractions of $A(R)$, namely, $D=(R \backslash\{0\})^{-1} A(R)$. We have $A^{\times}(R) / R^{\times}=\mathbf{G}\left(R_{0}\right)[18$, Proposition 4.9]. Let

$$
b=1-z^{-1} \in A^{\times}(R),
$$

and, for $u \in \mathbf{F}_{q^{3}}^{\times} \subset A^{\times}(R)$, set $b_{u}=u b u^{-1} \in A^{\times}(R)$. Let $\tilde{\Gamma}$ be the subgroup of $A^{\times}(R)$ generated by the elements $b_{u}$. Then $\Gamma$ is the quotient of $\tilde{\Gamma}$ modulo $R^{\times}$.

Write $X$ for the Bruhat-Tits building associated to $\operatorname{PGL}_{3}\left(\mathbf{F}_{q}((y))\right)$. The vertexes of $X$ are $\mathbf{F}_{q}[[y]]$-lattices in $\mathbf{F}_{q}((y))^{3}$, and incidence is given by flags. The action of $\mathbf{G}\left(R_{0}\right)$ on $X$ (via its embedding in $\mathrm{PGL}_{3}\left(\mathbf{F}_{q}((y))\right)$ ) is transitive on vertexes. The group $\Gamma$ is a normal subgroup of $\mathbf{G}\left(R_{0}\right)$ of finite index (see [7, Theorem 2.6]) which also acts transitively on the vertexes of $X$ (in fact, the reduced norm of $b$ is $y /(1+y)$, which coincide with $y$ up to the invertible element $1+y$ of $\left.\mathbf{F}_{q}[[y]]\right)$. The congruence subgroups of $\Gamma$ are defined as follows. Let $I$ be an ideal in $R$. The quotient map $R \rightarrow R / I$ induces an epimorphism $A(R) \rightarrow A(R / I)$, which itself induces a group morphism $A^{\times}(R) \rightarrow A^{\times}(R / I)$. Denote the kernel by $A^{\times}(R, I)$ and its quotient modulo $R^{\times}$by $\left(A^{\times} / R^{\times}\right)(R, I)$. 
Then we set $\Gamma(I)=\Gamma \cap\left(A^{\times} / R^{\times}\right)(R, I)$, and let $\Gamma_{I}=\Gamma / \Gamma(I)$ be the quotient group.

Let $f_{p} \in \mathbf{F}_{q}[y]$ be a monic irreducible polynomial prime to $y$ and $y+1$, let $s_{p} \geqslant 1$ be an integer, and let $I_{p}=\left\langle f_{p}^{s_{p}}\right\rangle$. The deterministic data for Theorem 1 is $\left(X, \Gamma,\left\{\Gamma\left(I_{p}\right)\right\}\right)$, where

- $X$ is the Bruhat-Tits building of $\operatorname{PGL}_{3}\left(\mathbf{F}_{q}((y))\right)$;

- $\Gamma$ is the Cartwright-Steger lattice in $\operatorname{PGL}_{3}\left(\mathbf{F}_{q}((y))\right)$;

- $\left\{\Gamma\left(I_{p}\right)\right\}$ are the arithmetic lattices associated with $I_{p}$.

We suppose that $\operatorname{deg}\left(f_{p}^{s_{p}}\right) \rightarrow \infty$.

The assertion that $\mathbb{Z}^{2} \hookrightarrow \Gamma$ for these random groups is related to the elementary algebraic structure of $\mathrm{PGL}_{3}$ and $\mathrm{PSL}_{3}$ over fields and local rings. As usual for a local ring $L, \mathrm{GL}_{3}(L)$ is the group of units of $\mathrm{M}_{3}(L), \mathrm{SL}_{3}(L)$ is the subgroup of $\mathrm{GL}_{3}(L)$ of matrices with determinant 1 , and $\mathrm{PGL}_{3}(L), \mathrm{PSL}_{3}(L)$ are the projective versions.

We start with a lemma. Let $(L, m)$ be a finite local principal ring of prime characteristic with residue field $\mathbf{F}_{q}=L / m$, uniformizer $\pi$, and length $s$ (the smallest integer $s>0$ such that $m^{s}=0$ ).

LEMMA 29. The order of the group $\left\langle\gamma, \gamma^{\prime}\right\rangle$ generated by two commuting elements $\gamma, \gamma^{\prime} \in \mathrm{PGL}_{3}(L)$ is at most

$$
3 q^{2\left\lceil\log _{q} s\right\rceil}\left(q^{3}-1\right),
$$

where $\log _{q}$ is the logarithm relative to $q$, and $\lceil\cdot\rceil$ is the upper integer value.

Proof. In the reduction modulo $m$

$$
\operatorname{PGL}_{3}(L) \ni \gamma \mapsto \bar{\gamma} \in \operatorname{PGL}_{3}\left(\mathbf{F}_{q}\right),
$$

an element $\gamma \in \mathrm{PGL}_{3}(L)$ such that $\bar{\gamma}=1$ has a representative of the form

$$
\lambda\left(1+\pi^{k} \gamma_{0}\right) \in \mathrm{GL}_{3}(L)
$$

for some $\lambda \in L^{\times}, \gamma_{0} \in \mathrm{M}_{3}(L)$, and $k \geqslant 1$. The Frobenius map gives

$$
\left(1+\pi^{k} \gamma_{0}\right)^{q^{\left[\log _{q} s\right\rceil}}=1,
$$

and therefore $\gamma^{q^{\left\lceil\log _{q} s\right\rceil}}=1$.

Thus, if $\gamma$ and $\gamma^{\prime}$ are two commuting elements in $\mathrm{PGL}_{3}(L)$, then the kernel $K$ in the exact sequence

$$
1 \rightarrow K \rightarrow\left\langle\gamma, \gamma^{\prime}\right\rangle \rightarrow\left\langle\bar{\gamma}, \bar{\gamma}^{\prime}\right\rangle \rightarrow 1
$$

is an abelian group of order at most $q^{2\left\lceil\log _{q} s\right\rceil}$. 
We now estimate $\left|\left\langle\bar{\gamma}, \bar{\gamma}^{\prime}\right\rangle\right|$. Assume without loss of generality that $L=\mathbf{F}_{q}$. Let $\gamma, \gamma^{\prime}$ be representatives of $\gamma, \gamma^{\prime}$ in $\mathrm{GL}_{3}(L)$, and let $f \in L[X]$ be the characteristic polynomial of $\gamma$.

Assume that $f$ does not have a root in $L$. Then $f$ is irreducible over $L$ (since a factorization would split off a degree- 1 factor) and is therefore equal to its minimal polynomial. It follows that the rational canonical form of $\gamma$ has only one block associated with $f$, and $\gamma$ has a cyclic vector $\xi$. Given $\gamma^{\prime} \in \operatorname{PGL}_{3}(L)$, there exists $P \in L[X]$ such that

$$
\underline{\gamma^{\prime}} \xi=P(\underline{\gamma}) \xi
$$

If

$$
\underline{\gamma} \underline{\gamma}^{\prime}=\lambda \underline{\gamma^{\prime}} \underline{\gamma}
$$

for some $\lambda \in L^{\times}$, then

$$
\underline{\gamma}^{k} \underline{\gamma}^{\prime}=\lambda^{k} \underline{\gamma}^{\prime} \underline{\gamma}^{k}
$$

so

$$
\underline{\gamma}^{\prime} \underline{\gamma}^{k} \xi=\lambda^{-k} \underline{\gamma}^{k} P(\underline{\gamma}) \xi=\lambda^{-k} P(\underline{\gamma}) \underline{\gamma}^{k} \xi
$$

Thus,

$$
\underline{\gamma^{\prime}}=P(\underline{\gamma})\left(\begin{array}{ccc}
1 & 0 & 0 \\
0 & \lambda^{-1} & 0 \\
0 & 0 & \lambda^{-2}
\end{array}\right)
$$

in the basis $\left(\xi, \underline{\gamma} \xi, \underline{\gamma}^{2} \xi\right)$. Taking determinants, the equality $\underline{\gamma} \underline{\gamma}^{\prime}=\lambda \underline{\gamma}^{\prime} \underline{\gamma}$ shows that $\lambda$ is a cube root of 1 . Thus every element of $\left\langle\underline{\gamma}, \underline{\gamma^{\prime}}\right\rangle$ belong to the subspace

$$
\{P(\underline{\gamma}) \mid P \in L[X]\}
$$

of $M_{3}(L)$, possibly after multiplying by an element of the form

$$
\left(\begin{array}{ccc}
1 & 0 & 0 \\
0 & \lambda & 0 \\
0 & 0 & \lambda^{2}
\end{array}\right)
$$

$\lambda \in L^{\times}$a cube root of 1 . Since the dimension of $\{P(\gamma) \mid P \in L[X]\}$ over $L$ is 3, the order of $\left\langle\underline{\gamma}, \underline{\gamma}^{\prime}\right\rangle$ is at most $3\left(q^{3}-1\right)$.

A similar argument works more generally if $f$ equals the minimal polynomial. Otherwise, $f$ has a root $a$ in $L$, and we may assume up to conjugacy that $\underline{\gamma}$ has the form $\left(\begin{array}{ll}a & 0 \\ 0 & \alpha\end{array}\right)$, where $a \in L^{\times}$and $\alpha \in \mathrm{GL}_{2}(L)$. Write

$$
\underline{\gamma^{\prime}}=\left(\begin{array}{ll}
b & m \\
n & \beta
\end{array}\right)
$$


$b \in L^{\times}, m^{t}, n \in L^{2}$, and $\beta \in \mathrm{GL}_{2}(L)$. Since

$$
\underline{\gamma \gamma^{\prime}}=\lambda \underline{\gamma}^{\prime} \underline{\gamma}
$$

for some $\lambda \in L^{\times}$, we have $a b=\lambda b a$, so

$$
\lambda=1,
$$

and thus $m \alpha=a m, \alpha n=a n$, and

$$
\alpha \beta=\beta \alpha .
$$

If $m=n=0$, then the argument of the previous section shows that the order of $\langle\alpha, \beta\rangle$ is at most $q^{2}-1$, so the order of $\left\langle\gamma, \gamma^{\prime}\right\rangle$ is at most $q^{3}-1$. Otherwise, $a$ is an eigenvalue of $\alpha$, and it is easy to see that the order of $\left\langle\gamma, \gamma^{\prime}\right\rangle$ is at most $q^{3}-1$ in this case too, which proves the lemma.

Proof of the assertion that $\mathbb{Z}^{2} \hookrightarrow \Gamma$ in Theorem 1. The local ring

$$
R / I_{p} \simeq \mathbf{F}_{q}[y] /\left\langle f_{p}(y)^{s_{p}}\right\rangle
$$

has residue field

$$
\mathbf{F}_{q_{p}}=R /\left\langle f_{p}\right\rangle \simeq \mathbf{F}_{q}[y] /\left\langle f_{p}(y)\right\rangle .
$$

By [18, Theorem 6.6],

$$
\operatorname{PSL}_{3}\left(R / I_{p}\right) \subset \Gamma_{I_{p}} \subset \operatorname{PGL}_{3}\left(R / I_{p}\right) .
$$

Let $\Lambda$ be any subgroup of $\Gamma$ isomorphic to $\mathbb{Z}^{2}$. Lemma 29 shows that the order of the image of $\Lambda$ in $\Gamma_{I_{p}}$ is at most

$$
3 q_{p}^{2\left\lceil\log _{q_{p}} s\right\rceil}\left(q_{p}^{3}-1\right)
$$

where $q_{p}$ is the order of the residue field. Denote by $\Pi \simeq \mathbb{R}^{2}$ the flat associated to $\Lambda$ in $X$, and let $\mathscr{C}_{p}$ be the set of $\Gamma\left(I_{p}\right)$-orbits of chambers in $X$. The set $F_{p}$ of $\Lambda \cap \Gamma\left(I_{p}\right)$ orbits of chambers in $\Pi$ is finite, and there is a constant $C$ (namely, $C=3 \times$ the number of $\Lambda$ orbits of chambers in $\Pi$ ) such that

$$
\left|F_{p}\right| \leqslant C q_{p}^{2\left\lceil\log _{q_{p}} s_{p}\right\rceil+3} .
$$

Let $F_{p}^{\prime}$ be the image of $F_{p}$ in $\mathscr{C}_{p}$.

Consider a sequence $Y_{1}, Y_{2}, Y_{3}, \ldots$ of independent identically distributed random variable with values in $\mathscr{C}_{p}$. The probability $\mathbb{P}\left(E_{n}\right)$ of the event,

$$
E_{n}=\left\{Y_{i} \notin F_{p}^{\prime}, \forall i \leqslant\left|\mathscr{C}_{p}\right|^{\delta}\right\},
$$


can be estimated by

$$
\mathbb{P}\left(E_{n}\right) \geqslant\left(1-\frac{\left|F_{p}^{\prime}\right|}{\left|\mathscr{C}_{p}\right|}\right)^{\left|\mathscr{C}_{p}\right|^{\delta}},
$$

and therefore

$$
\mathbb{P}\left(E_{n}\right) \geqslant e^{-2\left|\mathscr{C}_{p}\right|^{\delta-1}\left|F_{p}^{\prime}\right|} .
$$

Since $\Gamma_{I_{p}}$ acts freely on $\mathscr{C}_{p}$ with $\mathscr{C}_{p} / \Gamma_{I_{p}}$ fixed,

$$
\left|\mathscr{C}_{p}\right| \geqslant D\left|\Gamma_{I_{p}}\right|
$$

for some constant $D>0$. Let us compute $\left|\Gamma_{I_{p}}\right|$. The reduction map

$$
R / I_{p} \rightarrow R /\left\langle f_{p}\right\rangle=\mathbf{F}_{q_{p}}
$$

induces a surjective map

$$
\pi: \mathrm{GL}_{3}\left(R / I_{p}\right) \rightarrow \mathrm{GL}_{3}\left(\mathbf{F}_{q_{p}}\right)
$$

since det: $\mathrm{GL}_{3}\left(R / I_{p}\right) \rightarrow\left(R / I_{p}\right)^{\times}$commutes with reduction modulo $\left\langle f_{p}\right\rangle$, and since an element is invertible in $R / I_{p}$ if and only if its image in $\mathbf{F}_{q_{p}}$ is nonzero (as $R / I_{p}$ is local). Therefore any pull-back of a matrix of $\mathrm{GL}_{3}\left(\mathbf{F}_{q_{p}}\right)$ in $\mathrm{M}_{3}\left(R / I_{p}\right)$ is a matrix in $\mathrm{GL}_{3}\left(R / I_{p}\right)$. The kernel of $\pi$ consists of matrices of the form $\mathrm{Id}+\gamma_{0}$ where all coefficient of $\gamma_{0}$ belong to the ideal $\left\langle f_{p}\right\rangle$. Therefore

$$
\left|\mathrm{GL}_{3}\left(R / I_{p}\right)\right|=q_{p}^{9\left(s_{p}-1\right)}\left|\mathrm{GL}_{3}\left(\mathbf{F}_{q_{p}}\right)\right|=q_{p}^{9 s_{p}-6}\left(q_{p}^{3}-1\right)\left(q_{p}^{2}-1\right)\left(q_{p}-1\right) .
$$

As det is surjective and $a \in\left(R / I_{p}\right)^{\times}$if and only if $a$ is not a multiple of $f_{p}$, we have that

$$
\begin{aligned}
\left|\mathrm{SL}_{3}\left(R / I_{p}\right)\right| & =\frac{1}{\left|\left(R / I_{p}\right)^{\times}\right|}\left|\mathrm{GL}_{3}\left(R / I_{p}\right)\right| \\
& =\frac{1}{q_{p}^{s_{p}-1}\left(q_{p}-1\right)} q_{p}^{9 s_{p}-6}\left(q_{p}^{3}-1\right)\left(q_{p}^{2}-1\right)\left(q_{p}-1\right) \\
& =q_{p}^{8 s_{p}-5}\left(q_{p}^{3}-1\right)\left(q_{p}^{2}-1\right) .
\end{aligned}
$$

This gives

$$
\left|\Gamma_{I_{p}}\right| \geqslant\left|\mathrm{PSL}_{3}\left(R / I_{p}\right)\right|=\mu_{p}^{-1}\left|\mathrm{SL}_{3}\left(R / I_{p}\right)\right|=\mu_{p}^{-1} q_{p}^{8 s_{p}-5}\left(q_{p}^{3}-1\right)\left(q_{p}^{2}-1\right),
$$

where $\mu_{p}=\left|\left\{a \in\left(R / I_{p}\right)^{\times} \mid a^{3}=1\right\}\right|$. Therefore,

$$
\begin{aligned}
\left|\mathscr{C}_{n}\right|^{\delta-1}\left|F_{p}^{\prime}\right| & \leqslant C D^{\delta-1} \mu_{p}^{1-\delta} q_{p}^{2\left\lceil\log _{q_{p}} s_{p}\right\rceil+3} q_{p}^{8 s_{p}(\delta-1)} \\
& =C D^{\delta-1} \mu_{p}^{1-\delta} q_{p}^{2\left\lceil\log _{q_{p}} s_{p}\right\rceil+8 s_{p}(\delta-1)+3}
\end{aligned}
$$

Thus, since $\left\lceil\log _{q_{p}} s_{p}\right\rceil / s_{p} \longrightarrow_{n \rightarrow \infty} 0$ when $\operatorname{deg} f_{p}^{s_{p}} \rightarrow \infty$, we obtain the following. 
(i) If $s_{p}=1$ for large $n$, then $\left(R / I_{p}\right)^{\times}$is cyclic, $\mu_{p} \leqslant 3$ (for large $n$ ), and thus $\mathbb{P}\left(E_{n}\right) \rightarrow 1$ if

$$
8(\delta-1)+3<0
$$

that is,

$$
\delta<\frac{5}{8}
$$

(ii) If $s_{p} \geqslant k \geqslant 2$ for large $n$, then $\left(R / I_{p}\right)^{\times}$is not cyclic in general, but using the rough estimate $\mu_{p} \leqslant q_{p}^{s_{p}-1}$ (or $\mu_{p} \leqslant q_{p}-1$ if the order satisfy $q \neq 3^{\ell}$, $\ell=1,2,3, \ldots)$, we have that $\mathbb{P}\left(E_{n}\right) \rightarrow 1$ if

$$
(1-\delta)\left(s_{p}-1\right)+8 s_{p}(\delta-1)+3<0 ;
$$

that is,

$$
\delta<\frac{7 k-2}{7 k+1} .
$$

(iii) If $\left(f_{p}^{s_{p}}\right)$ does not satisfy (i) or (ii), we separate the cases $s_{p} \geqslant 2$ and $s_{p}=1$, and obtain the desired conclusion when $\delta<\frac{5}{8}$ as $(7 k-2) /(7 k+1)>\frac{5}{8}$ for $k \geqslant 2$.

This shows that $\mathbb{P}\left(E_{n}\right) \rightarrow 1$. Therefore, Lemma 11 applies with overwhelming probability, and we have that $\mathbb{Z}^{2} \hookrightarrow \Gamma$ with overwhelming probability.

REMARK 30. We have proved the stronger result that every embedding $\mathbb{Z}^{2} \hookrightarrow$ $\Gamma_{3}$ eventually lifts to an embedding $\mathbb{Z}^{2} \hookrightarrow \Gamma$ with overwhelming probability. Recall that, according to Mostow and Prasad, the set of embeddings $\mathbb{Z}^{2} \hookrightarrow \Gamma_{3}$ is dense [20].

QUESTION 31. Are these random groups residually finite? (For comparison, note that there exist nonresidually finite [8] central extensions of the form $1 \rightarrow \mathbb{Z} \rightarrow$ $\widehat{\operatorname{Sp}(2 n, \mathbb{Z})} \rightarrow \operatorname{Sp}(2 n, \mathbb{Z}) \rightarrow 1$. $)$

Question 32. What is the value of $\delta_{\mathbb{Z}^{2}}\left(X_{3}, \Gamma_{3},\left\{\Gamma_{3}\left(I_{p}\right)\right\}\right)$ ? What is the value of $\delta_{\mathbb{R}^{2}}\left(X_{3}, \Gamma_{3},\left\{\Gamma_{3}\left(I_{p}\right)\right\}\right)$ ?

QUESTION 33 ('Perforated buildings'). If $X$ is a Bruhat-Tits building of rank 2 (possibly exotic) and $Y \subset X$ is an $R$-separated subset of chambers, does $\mathbb{R}^{2} \hookrightarrow$ $X \backslash Y$ for $R$ large enough?

REMARK 34. It is proved in [18] that the congruence quotients of $X_{3}$ by $\Gamma_{3}\left(I_{p}\right)$ are Ramanujan complexes. This fact, whose proof relies on the 'JacquetLanglands correspondence' (in positive characteristic), is not used in this paper. 
(Nevertheless, the random quotients $X / \Gamma$ as in Theorem 1 are Ramanujan complexes 'up to $\varepsilon$ ', at least if the density is not too large.) The main input from algebraic groups used in the proof is Strong Approximation, and so it is most likely that results in the spirit of Theorem 1 can also be established for other algebraic groups (e.g. Sp) and over other fields (e.g. characteristic 0), the first step being to construct explicit families of lattices. We also note that the case of positive characteristic is easier to handle from an algorithmic perspective (the construction of $f_{p}$ can be done in polynomial time using the Shoup algorithm).

\section{Further properties of the random group}

The critical density $\delta_{T}\left(X, \Gamma,\left\{G_{p}\right\}\right)$ for Kazhdan's property T can be estimated using the so-called 'spectral criterion for property T', which is local. This is done by making (spectral) bounds on the local geometric perturbations explicit, following [10, Section 6] and [9].

We recall (see [5] for more details) that the 'spectral criterion' refers to the following well-known result of Garland [10, Theorem 5.9].

THEOREM 35 (Garland). Let $V$ be finite simplicial complex of dimension $\ell$ in which every simplex belongs to an $\ell$-cell, and let $r \leqslant \ell$. Assume that, for every vertex $v \in V^{(0)}$, the rth reduced cohomology group $\tilde{H}^{r}(\operatorname{Lk} v, \mathbb{R})$ [i.e. $\tilde{H}^{r}=H^{r}$ if $r \geqslant 1$ and $\left.\tilde{H}^{0}=\operatorname{coker}\left(H^{0}(\star) \rightarrow H^{0}\right)\right]$ of the link Lk $v$ of $V$ vanishes, and denote by $\lambda_{r}(\operatorname{Lk} v)$ the smallest nonzero eigenvalue of $\Delta^{+}=d \partial$ acting on $C^{r}(\operatorname{Lk} v)$. Set $\lambda_{r}:=\min _{v} \lambda_{r}(\mathrm{Lk} v)$. If

$$
\lambda_{r-1} \geqslant \frac{\ell-r}{r+1}
$$

then $H^{r}(V, \rho)=0$ for every finite-dimensional unitary representation $\rho$ of $\pi_{1}(V)$.

(If $\ell=2, r=1$, this says that

$$
\lambda_{0}>1 / 2 \Rightarrow H^{1}(V, \rho)=0
$$

for any $\rho$.

The statement in [10] deals only with those $V$ whose universal coverings are Bruhat-Tits buildings, but Borel points out [6, Theorem 1.5] that Garland's argument is general. (While we are mostly interested in Bruhat-Tits buildings here, it is the general case that is useful for us.) Borel also notes in Section 2.2 that the assumption that $V$ is finite can be replaced by 'uniformly locally finite', provided that the conclusion refers to the vanishing of $L^{2} H^{r}(V, \rho)\left(L^{2}\right.$-summable cochains). It was later shown that the family of admissible $\rho$ with vanishing $H^{r}$ could be substantially enlarged; in particular, the linearity assumption on $\rho$ can 
be disposed of (in the smooth category [24]), as can the finite-dimensionality assumption on $\rho$ (see $[1,23,25])$. The latter leads to the conclusion that $H^{r}(V$, $\rho)=0$ for every unitary representation $\rho$ of $\pi_{1}(V)$ which, when $r=1$, is equivalent to $\Gamma$ having Kazhdan's property T by a well-known result of Delorme and Guichardet (see [5]). Garland studies in particular the two-dimensional case, exploiting the computation of the exact value of $\lambda_{0}$ for Tits buildings (associated with BN-pairs, the computation appears in [9] and [10, Proposition 7.10]) to derive the vanishing of $H^{1}$ 'when the order is large enough'. (As Garland observes, his cohomology vanishing results for lattices-with finite-dimensional unitary targets-were in fact already known in degree 1 , as they were covered by Kazhdan's work.)

We now explain why property $\mathrm{T}$ holds for random groups 'when the order is large enough' (in particular, when $q \geqslant 5$ if $\delta<1 / 2$, and the deterministic data is of type $\tilde{A}_{2}$, and for $q \gg 1$ if $\delta$ is only assumed to be bounded away from 1).

The idea is that $\lambda_{0}$ is controlled if the number of chambers removed is significantly smaller than the order (defined as the minimal number of chambers on an edge minus 1) of $V$.

LEMMA 36. For every $\delta>0$ and every integer $n \geqslant 0$, there exists a constant $\delta^{\prime}>0$ such that, if $G$ is a graph of order $q \geqslant 5 n / \delta$ with $\lambda_{0}(G)>1-\delta^{\prime}$, and $G^{\prime} \subset G$ is a subgraph with $\left|G^{(1)}\right| \geqslant\left|G^{(1)}\right|-n$, then $\lambda_{0}\left(G^{\prime}\right) \geqslant 1-\delta$.

Indeed, by the Cheeger inequality (for graphs), it is enough to prove the corresponding statement for the Cheeger constant; then the numerators of the two isoperimetric ratios differ by a constant that is washed out as $q \rightarrow \infty$.

More explicitly, we have the following.

Proof. For a subset $A \subset V$, let $h_{G}(A):=\left|\partial_{G} A\right| /\left(\min \left(|A|_{G},|X-A|_{G}\right)\right)$, where $\left|\partial_{G} A\right|$ is the number of edges with extremities belonging to both $A$ and $X-A$, and $|A|_{G}:=\sum_{x \in A} \operatorname{val}_{G}(x)$. The Cheeger constant of $G$ is $h(G):=\min _{A \subset G} h_{G}(A)$. Let $A$ be a subset of $V^{\prime}$ such that $h\left(G^{\prime}\right)=h(A)$. By the Cheeger inequality,

$$
\begin{aligned}
\lambda_{0}(G) \leqslant 2 h(G) & \leqslant \frac{2\left|\partial_{G} A\right|}{\min \left(|A|_{G},|X-A|_{G}\right)} \\
& \leqslant \frac{2\left|\partial_{G^{\prime}} A\right|+2 n}{\min \left(|A|_{G^{\prime}},|X-A|_{G^{\prime}}\right)}=2 h\left(G^{\prime}\right)+\frac{2 n}{\min \left(|A|_{G^{\prime}},|X-A|_{G^{\prime}}\right)} .
\end{aligned}
$$

Since $q \geqslant 5 n / \delta$, we obtain $\lambda_{0}(G) \leqslant 2 h\left(G^{\prime}\right)+\delta / 2$. Thus

$$
\lambda_{0}(G) \leqslant 2 \sqrt{2 \lambda_{0}\left(G^{\prime}\right)}+\delta / 2,
$$

using again the Cheeger inequality. 
Let $X$ be a thick irreducible Euclidean building of rank 2. The links of $X$ are spherical buildings, and there exist integers $q^{*} \geqslant q_{*} \geqslant 2$ such that the degree of an edge in $X$ is either $q^{*}+1$ or $q_{*}+1$. We call $q_{*}$ the order of $X$. In the $\tilde{A}_{2}$ case, $q^{*}=q_{*}$.

THEOREM 37 (Generic property T). For every $\delta_{0}<1$, there exists $q_{0}$ such that, if $\delta<\delta_{0}$ and if in the deterministic data $\left(X, \Gamma,\left\{\Gamma_{p}\right\}\right)$, the space $X$ is a thick classical (i.e. associated with an algebraic group over a local field) irreducible Bruhat-Tits building of rank 2 and order $q_{*} \geqslant q_{0}$, then the random group at density $\delta$ with deterministic data $\left(X, \Gamma,\left\{\Gamma_{p}\right\}\right)$ has Kazhdan's property $T$ with overwhelming probability. Thus

$$
\delta_{T}\left(X, \Gamma,\left\{\Gamma_{p}\right\}\right) \rightarrow 1
$$

as $q_{*}(X) \rightarrow \infty$.

REMARK 38. The groups appearing in the above result (and in Corollary 17) are buildings with chambers missing in the sense of [3]. Some (weak) buildings with chambers missing have the 'opposite' Haagerup property; see [3]. We also note that random groups in the density model admit an infinite quotient with property $\mathrm{T}$, independently of the order, as soon as we are given deterministic data with property $\mathrm{T}$.

Proof. Let $k$ be sufficiently large that $\delta_{0}<k /(k+1)$. Let $0<\varepsilon<1 / 2$ be given. By Lemma 36, we can find $\varepsilon^{\prime}>0$ such that, if $\lambda_{0}(G)>1-\varepsilon^{\prime}$, then $\lambda_{0}(G-k)>$ $1-\varepsilon>1 / 2$. Feit and Higman [9] and Garland [10] show that, if $G_{q}$ is a spherical building of order $q$, then $\lambda_{0}\left(G_{q}\right)$ converges to 1 as $q \rightarrow \infty$. Let $q_{0}$ be larger than $10 k$ and be such that $\lambda_{0}\left(G_{q}\right)>1-\varepsilon^{\prime}$ for any spherical building $G_{1}$ of order $q \geqslant q_{0}$. If, in the deterministic data $\left(X, \Gamma,\left\{\Gamma_{p}\right\}\right)$, the space $X$ is a thick classical irreducible building of rank 2 such that $q_{*}(X) \geqslant q_{0}$, and if $\delta<\delta_{0}$, then, by Proposition 27, the links of $X$ contain at most $k$ chambers missing at every vertex, with overwhelming probability. Therefore, the random perforated building $X \backslash \Gamma A$ at density $\delta$ satisfies $\lambda_{0}(X \backslash \Gamma A) \geqslant 1-\varepsilon>1 / 2$ with overwhelming probability. Since the random universal cover $\widehat{X \backslash \Gamma A}$ is locally isomorphic to $X \backslash \Gamma A$, Theorem 35 applies. Thus the random group has property $\mathrm{T}$ (and the spectral gap is arbitrarily close to 1 ) with overwhelming probability.

It is natural to wonder if the bound on the order can be estimated. It seems difficult to give a general answer, but we can do it at least when the density is $<1 / 2$ (or in the model with few chambers missing). In fact, one can compute the exact value of $\lambda_{0}(X)$, in the spirit of Feit and Higman's paper [9]. 
More precisely, we have the following.

THEOREM 39. If $\left(X, \Gamma,\left\{\Gamma_{p}\right\}\right)$ is of type $\tilde{A}_{2}$, then, both in the bounded model and in the density model of parameter $\delta<1 / 2$, the random group has property $T$ (with overwhelming probability) when $q \geqslant 5$.

This follows from the following.

Proposition 40. Let $G$ be a spherical building of type $A_{2}$ and order $q$ with a single chamber missing. Then

$$
\lambda_{0}(G)=1-\frac{\sqrt{q+1 / 4}+1 / 2}{q+1} .
$$

In particular, $\lambda_{0}(G)>1 / 2$ whenever $q \geqslant 5$.

We recall (see, e.g., [5]) that the value computed by Feit and Higman in the $A_{2}$ case is

$$
\lambda_{0}(G)=1-\frac{\sqrt{q}}{q+1}
$$

so, in particular, $\lambda_{0}(G)>1 / 2$ whenever $q \geqslant 2$.

Proof. Let $G \leadsto G^{\prime}$ be an extension into an $A_{2}$ building of order $q$ (see [3]), and let $P$ be the projective plane corresponding to $G^{\prime}$. We choose the following basis for the space of functions on the vertex set of $G$ :

$$
\left(\delta_{p_{1}}, \delta_{p_{2}}, \ldots \delta_{p_{q^{2}+q+1}}, \delta_{l_{1}}, \delta_{l_{2}}, \ldots, \delta_{l_{q^{2}+q+1}}\right),
$$

where $p_{1} \in G$ (respectively $l_{1} \in G$ ) corresponds to the point $p$ (respectively, the line $l$ ) of $P$ associated to the missing chamber, while $p_{2}, \ldots, p_{q+1}$ (respectively, $\left.l_{2}, \ldots, l_{q+1}\right)$ correspond to an enumeration of the points of $l$ distinct from $p$ (respectively, the lines adjacent to $p$ distinct from $l$ ) in $P$.

The Laplace operator $\Delta$ is of the form

$$
\Delta=\mathrm{Id}-\left(\begin{array}{cc}
0 & A \\
A^{t} & 0
\end{array}\right)
$$

where $A$ is the normalized adjacency matrix, namely $A=D_{q}^{-1 / 2} A_{0} D_{q}^{-1 / 2}$, where $A_{0}$ is the usual (bipartite) adjacency matrix (we recall that $\left(A_{0}\right)_{i, j}=1$ when $l_{i}$ is adjacent to $p_{j}$ ), and $D_{q}$ is the diagonal matrix having $q+1$ down the diagonal, except on the first entry which is $q$. Denote $\tilde{q}=\sqrt{q(q+1)}$. A computation shows 
that

$$
A A^{t}=A^{t} A=\left(\begin{array}{ccc}
q \tilde{q}^{-2} & 0_{q} & (q+1)^{-1} \tilde{q}^{-1} \mathbf{1}_{1 \times q^{2}} \\
0_{q} & B_{q} & (q+1)^{-2} \mathbf{1}_{q \times q^{2}} \\
(q+1)^{-1} \tilde{q}^{-1} \mathbf{1}_{q^{2} \times 1} & (q+1)^{-2} \mathbf{1}_{q^{2} \times q} & C_{q^{2}}
\end{array}\right)
$$

where $B_{q}$ is the $q \times q$ matrix with

$$
\frac{q}{(q+1)^{2}}+\frac{1}{q(q+1)}
$$

on the diagonal, and $(q-1) / q(q+1)$ elsewhere, while $C_{q^{2}}$ is the $q^{2} \times q^{2}$ matrix with $(q+1)^{-1}$ on the diagonal and $(q+1)^{-2}$ elsewhere. Set

$$
\gamma_{q}=(q+1) \tilde{q}^{-1}=\sqrt{1+\frac{1}{q}}
$$

We have

$$
(q+1)^{2} A^{t} A-q \mathrm{Id}=\left(\begin{array}{ccc}
1 & 0_{q} & \gamma_{q} \mathbf{1}_{1 \times q^{2}} \\
0_{q} & \gamma_{q}^{2} \mathbf{1}_{q \times q} & \mathbf{1}_{q \times q^{2}} \\
\gamma_{q} \mathbf{1}_{q^{2} \times 1} & \mathbf{1}_{q^{2} \times q} & \mathbf{1}_{q^{2} \times q^{2}}
\end{array}\right)
$$

whose kernel is of codimension 3 . Consider the vectors

$$
v_{1}=\left(\begin{array}{c}
1 \\
0_{q} \\
\gamma_{q} \mathbf{1}_{q^{2} \times 1}
\end{array}\right) v_{2}=\left(\begin{array}{c}
0 \\
\gamma_{q}^{2} \mathbf{1}_{q \times 1} \\
\mathbf{1}_{q^{2} \times 1}
\end{array}\right) v_{3}=\left(\begin{array}{c}
\gamma_{q} \\
\mathbf{1}_{q^{2}+q \times 1}
\end{array}\right)
$$

A direct computation shows that

$$
\begin{aligned}
& (q+1)^{2} A^{t} A v_{1}=(q+1) v_{1}+q^{2} \gamma_{q} v_{3} \\
& (q+1)^{2} A^{t} A v_{2}=\left(q+q \gamma_{q}^{2}\right) v_{2}+q^{2} v_{3} \\
& (q+1)^{2} A^{t} A v_{3}=\gamma_{q} v_{1}+q v_{2}+\left(q^{2}+q\right) v_{3}
\end{aligned}
$$

Thus the problem reduces to computing the spectrum of the $3 \times 3$ matrix

$$
\left(\begin{array}{ccc}
q+1 & 0 & \gamma_{q} \\
0 & 2 q+1 & q \\
q^{2} \gamma_{q} & q^{2} & q^{2}+q
\end{array}\right)
$$

The characteristic polynomial is

$$
-x^{3}+\left(q^{2}+4 q+2\right) x^{2}-\left(2 q^{3}+6 q^{2}+4 q+1\right) x+q^{2}(q+1)^{2},
$$


where $(q+1)^{2}$ is an obvious root. The two other roots are

$$
x=(2 q+1-\sqrt{4 q+1}) / 2
$$

and

$$
x=(2 q+1+\sqrt{4 q+1}) / 2 .
$$

Therefore, the eigenvalue of $\left(\begin{array}{cc}0 & A \\ A^{t} & 0\end{array}\right)$ we are interested in is

$$
\frac{1}{q+1} \sqrt{q+\frac{1}{2}+\sqrt{q+\frac{1}{4}}}=\frac{\frac{1}{2}+\sqrt{q+\frac{1}{4}}}{q+1} .
$$

A similar computation can be worked out when two chambers are missing (arguing according to the respective positions of the chambers in an apartment), but the value of

$$
\lambda_{0, k}(G):=\inf _{E \subset G^{(1)},|E|=k} \lambda_{0}(G \backslash E)
$$

when $G$ is a spherical building (of dimension 1) might be more challenging to find. It would also be interesting to make similar estimates with more general (e.g. smooth) targets $\lambda_{0, k}(G, Y)$ and deduce the corresponding fixed points theorem for the random groups following [17, 24].

The results in [3] on buildings with chambers missing and [2] on property RD can be applied to our random groups in the $\tilde{A}_{2}$ case. This shows in particular that the random group is rigid in the sense that it remembers the building that it comes from (see [3, Section 5]). Furthermore, by [2, Corollary 6], the random group satisfies the Baum-Connes conjecture without coefficients.

THEOREM 41. If the deterministic data is of type $\tilde{A}_{2}$, then the following hold.

(1) The random group $(\Gamma, X)$ at density $\delta<\frac{1}{2}$ admits the unique extension $(\Gamma$, $X) \leadsto\left(\Gamma^{\prime}, X^{\prime}\right)$ (in the sense of [3, Section 1]) into a Euclidean building.

(2) The random group $\Gamma$ at arbitrary density satisfies the Baum-Connes conjecture without coefficients.

The first assertion is a consequence of Proposition 26 and [3, Theorem 5.11]. It is unknown if $\Gamma$ satisfies the Baum-Connes conjecture with coefficients. The conjecture without coefficients is also unknown for the other Coxeter types (in the irreducible case), but would follow from the 'interpolation of property RD to intermediate rank situations' (see [2]), that is, between hyperbolic groups and (uniform) higher rank lattices of the corresponding type, provided that the latter groups satisfy property RD (which is conjectured by Valette). 


\section{Acknowledgements}

We are grateful to the referees for helpful comments on the text.

The second author was supported by NSERC Discovery Grant 418144-12 and FQRNT NC Grant 166037.

\section{References}

[1] W. Ballmann and J. Swiatkowski, 'On $L^{2}$-cohomology and property (T) for automorphism groups of polyhedral cell complexes', Geom. Funct. Anal. 7 (4) (1997), 615-645.

[2] S. Barré and M. Pichot, 'Intermediate rank and property RD', arXiv:0710.1514.

[3] S. Barré and M. Pichot, 'Removing chambers in Bruhat-Tits buildings', Israel J. Math. (accepted), arXiv:1003.4614.

[4] S. Barré and M. Pichot, 'An exotic group with the Haagerup property', arXiv:1205.1128.

[5] B. Bekka and P. de la Harpe, Valette, Alain Kazhdan's Property (T), New Mathematical Monographs, 11, (Cambridge University Press, Cambridge, 2008).

[6] A. Borel, Cohomologie de certains groupes discrets et laplaciens p-adiques [d'après H. Garland], Séminaire Bourbaki, exposé no 437, 1973.

[7] D. I. Cartwright and T. Steger, 'A family of $\tilde{A}_{n}$-groups', Israel J. Math. 103 (1998), 125-140.

[8] P. Deligne, 'Extensions centrales non résiduellement finies de groupes arithmétiques', $C$. $R$. Acad. Sci. Paris (1978).

[9] W. Feit and G. Higman, 'The nonexistence of certain generalized polygons', J. Algebra 1 (1964), 114-131.

[10] H. Garland, 'p-adic curvature and the cohomology of discrete subgroups of p-adic groups', Ann. of Math. t. 97 (1973), 375-423.

[11] Ghys, Étienne Groupes aléatoires (d'après Misha Gromov, ...) Astérisque No. 294 (2004), viii, 173-204.

[12] M. Gromov, 'Hyperbolic manifolds, groups and actions', in Riemann Surfaces and Related Topics: Riemann Surfaces and Related Topics, Proceedings of the 1978 Stony Brook Conference (1978).

[13] M. Gromov, 'Infinite groups as geometric objects', in Proc. Int. Congress Math. Warsaw 1983, Vol. 1 (1984), 385-392.

[14] M. Gromov, 'Hyperbolic groups', in Essays in Group Theory, Mathematical Sciences Research Institute Publications, 8 (Springer, New York, 1987), 75-263.

[15] M. Gromov, Geometric Group Theory, Vol. 2, Proceedings of the Symposium Held at Sussex University, Sussex, July 1991, (eds G. A. Niblo and M. A. Roller), London Mathematical Society Lecture Note Series, 182 (Cambridge University Press, Cambridge, 1993).

[16] M. Gromov, Spaces and questions (Tel Aviv, 1999), Geom. Funct. Anal. Special Volume (2000), Part I, 118-161.

[17] M. Gromov, Random walk in random groups, preprint IHES (2001), GAFA, Geom. Funct. Anal., Vol. 13 (2003).

[18] A. Lubotzky, B. Samuels and U. Vishne, 'Explicit constructions of Ramanujan complexes of type $\tilde{A}_{d}$ ', European J. Combin. 26 (6) (2005), 965-993 (English summary).

[19] G. A. Margulis, 'Discrete subgroups of semisimple Lie groups', in Ergebnisse der Mathematik und ihrer Grenzgebiete (3), [Results in Mathematics and Related Areas (3)] 17 (Springer-Verlag, Berlin, 1991). 
[20] G. D. Mostow, 'Strong rigidity of locally symmetric spaces', Annals of Mathematics Studies, 78 (Princeton University Press, Princeton, NJ, 1973), University of Tokyo Press, Tokyo.

[21] Y. Ollivier, 'A January 2005 invitation to random groups', in Ensaios Matemticos [Mathematical Surveys], 10 (Sociedade Brasileira de Matematica, Rio de Janeiro, 2005), (See also 'January 2010 random groups updates').

[22] Y. Ollivier, 'Sharp phase transition theorems for hyperbolicity of random groups', GAFA, Geom. Funct. Anal. 14 (3) (2004), 595-679.

[23] P. Pansu, 'Formules de Matsushima, de Garland et proprit (T) pour des groupes agissant sur des espaces symtriques ou des immeubles', Bull. Soc. Math. France 126 (1998), 107-139.

[24] M. T. Wang, 'A fixed point theorem of discrete group actions on Riemannian manifolds', J. Differential Geom. 50 (2) (1998), 249-267.

[25] A. Żuk, 'La propriété (T) pour les groupes agissant sur les polyèdres', C. R. Acad. Sci. Paris, t. 323 (1996), 453-458. 